\title{
Deformations of the circular Wilson loop and spectral (in)dependence
}

\author{
Michael Cooke, ${ }^{a}$ Amit Dekel, ${ }^{b}$ Nadav Drukker, ${ }^{a}$ Diego Trancanelli $^{c}$ and \\ Edoardo Vescovi ${ }^{c, d}$ \\ ${ }^{a}$ Department of Mathematics, King's College London, \\ The Strand, WC2R 2LS London, United Kingdom \\ ${ }^{b}$ Nordita, KTH Royal Institute of Technology and Stockholm University, \\ Roslagstullsbacken 23, SE-106 91 Stockholm, Sweden \\ ${ }^{c}$ Institute of Physics, University of São Paulo, \\ 05314-970 São Paulo, Brazil \\ ${ }^{d}$ The Blackett Laboratory, Imperial College, \\ London SW' 2AZ, United Kingdom \\ E-mail: cookepm@tcd.ie, amit.dekel@nordita.org, \\ nadav.drukker@gmail.com, dtrancan@if.usp.br, e.vescovi@imperial.ac.uk
}

ABSTRACT: In this paper we study the expectation value of deformations of the circular Wilson loop in $\mathcal{N}=4$ super Yang-Mills theory. The leading order deformation, known as the Bremsstrahlung function, can be obtained exactly from supersymmetric localization, so our focus is on deformations at higher orders. We find simple expressions for the expectation values for generic deformations at the quartic order at one-loop at weak coupling and at leading order at strong coupling. We also present a very simple algorithm (not requiring integration) to evaluate the two-loop result. We find that an exact symmetry of the strong coupling sigma-model, known as the spectral-parameter independence, is an approximate symmetry at weak coupling, modifying the expectation value starting only at the sextic order in the deformation. Furthermore, we find very simple patterns for how the spectral parameter can appear in the weak coupling calculation, suggesting all-order structures.

Keywords: Wilson, 't Hooft and Polyakov loops, AdS-CFT Correspondence, Conformal and W Symmetry, Integrable Field Theories

ArXiv EPrint: 1811.09638 


\section{Contents}

1 Introduction 1

2 From world-sheet to Wilson loop 4

2.1 Perturbations around the circle 6

3 Expectation value of perturbed circle $\quad 9$

3.1 One-loop order 9

3.2 Two-loop order 11

4 Spectral dependence at higher orders in $\epsilon \quad 13$

$\begin{array}{lll}5 & \text { Strong coupling } & 14\end{array}$

$\begin{array}{llr}6 & \text { Discussion } & 14\end{array}$

$\begin{array}{lr}\text { A Two-loop integrals } & 16\end{array}$

$\begin{array}{lll}\text { A.1 Logarithmic integrals } & 16\end{array}$

$\begin{array}{lll}\text { A.2 Non-logarithmic part in two-loop interacting diagrams } & 18\end{array}$

$\begin{array}{lll}\text { A.3 Two-loop ladder diagrams } & 19\end{array}$

\section{Introduction}

Wilson loop operators are among the most interesting observables that can be studied in gauge theories. For $\mathcal{N}=4$ super Yang-Mills (SYM) theory in four dimensions, these operators are supported along a contour in spacetime and may couple to the scalar fields $\Phi^{I}$ in the gauge multiplet. The most symmetric (and supersymmetric) operator is the $1 / 2$ BPS circle

$$
W=\frac{1}{N} \operatorname{Tr} \mathcal{P} \exp \oint d \theta\left(i A_{\mu} \dot{x}^{\mu}(\theta)+|\dot{x}| \Phi^{1}\right), \quad x^{\mu}(\theta)=(\cos \theta, \sin \theta, 0,0) .
$$

The exact vacuum expectation value (VEV) of the $1 / 2$ BPS circle is given by Laguerre polynomials, which reduce to a Bessel function in the large $N$ limit [1-3]

$$
\langle W\rangle=\frac{1}{N} L_{N-1}^{1}(\lambda / 4 N) e^{\lambda / 8 N}=\frac{2}{\sqrt{\lambda}} I_{1}(\sqrt{\lambda})+\mathcal{O}\left(1 / N^{2}\right) .
$$

This paper is concerned with non-supersymmetric deformations of this operator, where the contour is still restricted to be inside the same Euclidean plane of the circle and 
with the same constant scalar coupling. The contour is represented by the function (and Fourier series)

$$
X(\theta)=x^{1}(\theta)+i x^{2}(\theta)=e^{i \theta+g(\theta)}, \quad g(\theta)=\sum_{n=-\infty}^{\infty} b_{n} e^{i n \theta} .
$$

Clearly, for $g(\theta)=0$ we recover the circle, so small deformations can be written as a power series in $b_{n}$. For infinitesimal $b_{n}$ 's, neither self-intersections nor cusps form, and, consequently, no divergences appear in the evaluation of the expectation values. We restrict our attention to the planar limit, mainly to allow for comparisons with the strong coupling results of [4].

The order $\mathcal{O}\left(b_{n}^{2}\right)$ has already been studied in the past and is known as the wavy-circle (or wavy-line) approximation [5]. It turns out that, at this order, the coupling dependence of the expectation value of these Wilson loops is given by the Bremsstrahlung function $B(\lambda)$ introduced in [6]. Evaluating the one-loop order in $\lambda$ (or the classical strong coupling limit, using holography) is sufficient to determine this expectation value to all orders in perturbation theory [7]

$$
\frac{\left.\langle W\rangle\right|_{b^{2}}}{\left.\langle W\rangle\right|_{b^{0}}}=\pi^{2} B(\lambda) \sum_{n=2}^{\infty} n\left(n^{2}-1\right)\left|b_{n}+\bar{b}_{-n}\right|^{2}, \quad B(\lambda)=\frac{1}{4 \pi^{2}} \frac{\sqrt{\lambda} I_{2}(\sqrt{\lambda})}{I_{1}(\sqrt{\lambda})}
$$

where $\left.\langle W\rangle\right|_{b^{0}}$ is the expression in (1.2). This vanishes for $b_{-n}=-\bar{b}_{n}$, which corresponds to the case of an imaginary $g(\theta)$ and is just a reparametrization of the curve. Assuming $b_{-n}=\bar{b}_{n}$ and using (1.2), this can be written as

$$
\left.\langle W\rangle\right|_{b^{2}}=2 I_{2}(\sqrt{\lambda}) \sum_{n=2}^{\infty} n\left(n^{2}-1\right)\left|b_{n}\right|^{2}
$$

These formulas have been previously written as integrals over $g(\theta)$, and this is their Fourier representation. This transformation is done explicitly in the one-loop calculation in section 3.1 below. Indeed, some of the main calculations that we undertake in this paper are to evaluate one- and two-loop integrals in the Fourier basis, which is a natural representation of smooth deformations of the circular Wilson loop.

Much less is known about the corrections beyond the wavy approximation. Going to higher orders in $b_{n}$, one can see that there is no contribution at cubic order thanks to conformal symmetry. The first nontrivial order is therefore quartic in $b_{n}$, which is the focus of this paper. We find closed-form expressions at this deformation order for both one-loop in perturbation theory and leading classical order at strong coupling. We also present an efficient algorithm to calculate the exact $\mathcal{O}\left(\lambda^{2}\right)$ result for any loop specified by a finite set of non-zero $b_{n}$ 's.

Note that there are several natural other ways to represent deformations of the circular Wilson loop. One is in terms of local "bumps", which can be reinterpreted as the insertion of local operators into the circle and were the subject of recent analyses in [8-11]. Another parametrization arises in the evaluation of the expectation value of Wilson loops at strong coupling, via holography. It is well-known that this evaluation consists in finding the 
(regularized) area of a minimal string surface anchored to the Wilson loop's contour on the $A d S_{5}$ boundary. In the case of Wilson loops confined to a plane, the dual string world-sheet is contained in an Euclidean $A d S_{3}$ and there is a standard method, reviewed in the next section, to find such surfaces through the Pohlmeyer reduction of the bosonic string sigma-model $[4,12]$. In this representation, the string world-sheet (and hence the boundary contour) is determined by a holomorphic function $f(z)$. For the $1 / 2$ BPS circle, this function vanishes, so one can parametrize deformations of the circle in terms of ${ }^{1}$

$$
f(z)=\epsilon \sum_{p=2}^{\infty} a_{p} z^{p-2},
$$

with small $\epsilon$. The map between the two descriptions (1.3) and (1.6) was studied in great detail in [13] and is reviewed below in section 2. In that paper, the map was established between particular examples of deformations of the circle and the function $f(z)$ to high orders in the $\epsilon$-expansion. Here we consider the most general deformation, but focus mainly on the order $\epsilon^{4}$. As reviewed below, the map between the two descriptions, albeit complicated, is explicit. Since this map is non-linear, results at a certain order in the coefficients $b_{n}$ in (1.3) end up including higher powers of $\epsilon$.

One interesting property of this last representation of the Wilson loops is that the classical action of the string does not depend on the overall phase of $f(z)$, so it is invariant under $f(z) \rightarrow e^{i \varphi} f(z) .^{2}$ Indeed $\varphi$ is the spectral parameter in the integrability description of the string sigma-model and parametrizes the "master" symmetry used to construct an infinite number of non-local Yangian charges in $[14,15]$.

One of the main motivations for this work is to address a puzzle raised in [13]. ${ }^{3}$ The shape of the string world-sheet and of the Wilson loop contour depends on $\varphi$, and indeed the expectation value of the Wilson loop may depend on $\varphi$ beyond the classical string limit, and in particular at weak coupling in the gauge theory. In the examples studied in [13], there was indeed such dependence on $\varphi$ at one-loop order in $\lambda$. This dependence, however, did not appear at orders $\epsilon^{4}$ and $\epsilon^{6}$ in the deformation around the circle. This dependence appeared only at higher orders in $\epsilon$ (either $\epsilon^{8}$ or $\epsilon^{16}$ depending on the specific curve), showing that spectral transformations are an unexpected approximate symmetry at weak coupling. The main goal of this paper is to make this previous analysis more systematic and extend the weak-coupling computation to two-loops. We indeed confirm that there is no $\varphi$-dependence at order $\epsilon^{4}$ in the one-loop expectation value of deformed circles. The same conclusion holds at two-loops for any $f(z)$ polynomials of degree equal or less than 10 and we conjecture that it is true for arbitrarily high degree. By performing a more comprehensive survey, we find that generically there is $\varphi$-dependence at order $\epsilon^{6}$ in

\footnotetext{
${ }^{1}$ We adopt this peculiar mismatch between the subscript of $a$ and the power of $z$, as it makes many subsequent formulas significantly simpler.

${ }^{2}$ One can choose the phase by taking $f(1)$ to be real or the first nonzero $a_{p}$ to be real, but fixing such a choice is not really necessary.

${ }^{3}$ The spectral parameter was called $\lambda=e^{i \varphi}$ in [13] and the corresponding transformations were referred to as $\lambda$-deformations. We do not use this terminology here to avoid potential confusion with the 't Hooft coupling.
} 
the one-loop expectation value. The absence of this dependence in [13] is particular to the examples studied there.

In proving all this, we set up an algorithm computing the Wilson loop's expectation value on a deformed circle at one- and two-loops in $\lambda$ and up to order $\epsilon^{4}$. The output is a polynomial of Fourier coefficients $b_{n}$ in (1.3) or of the Taylor coefficients $a_{p}$ in (1.6). The one-loop expression is in closed form and can be generalized to higher orders in $\epsilon$. The two-loop results are expressed as recurrence relations. The calculation does not involve numerical approximations and it is more efficient than evaluating multiple integrals [16] when the loop is known in Fourier series representation around the circle (1.3) or in terms of $f(z)$.

The paper is organized as follows. In section 2 we summarize the approach of [4] for constructing minimal surfaces in Euclidean $A d S_{3}$ and their boundary curves from $f(z)$ and focus in section 2.1 on small deformations of the circular curve. Section 3 contains the main result of this paper: the one-loop and two-loop expectation values at order $\epsilon^{2}$ and $\epsilon^{4}$ and the spectral independence to order $\epsilon^{4}$. In section 4 we discuss the spectral dependence at order $\epsilon^{6}$ and beyond. We comment on interesting patterns in the expectation value at classical order at strong coupling in section 5. We present some conclusions and discussions in section 6. Details on the perturbative algorithm are relegated to an appendix.

\section{From world-sheet to Wilson loop}

In this section we recount the construction of Wilson loops from the Pohlmeyer description of Euclidean $A d S_{3}[4,17]$, discuss the spectral parameter deformation, and focus on the case of nearly circular Wilson loops.

For the string world-sheet we take the unit disk with complex coordinate $z=r e^{i \theta}$. The three-dimensional target space can be parametrized by a complex $X$ and a real $Z$, such that the boundary of $A d S$ is at $Z=0$, which is also the boundary of the world-sheet, $r=1$. So, at $r=1$ we have a curve $X(\theta)$, which is the boundary curve of the string, i.e., the Wilson loop contour.

Using the Pohlmeyer reduction of the sigma-model, a solution of the string equations of motion is characterized by a holomorphic function $f(z)$. From it, one constructs a real function $\alpha(z, \bar{z})$ solving the generalized cosh-Gordon equation

$$
\partial \bar{\partial} \alpha(z, \bar{z})=e^{2 \alpha(z, \bar{z})}+|f(z)|^{2} e^{-2 \alpha(z, \bar{z})} .
$$

The regularized area of the world-sheet, which equals the expectation value of the Wilson loop at strong coupling, then evaluates to

$$
A_{\text {reg }}=-2 \pi \sqrt{\lambda}-2 \sqrt{\lambda} \int_{|z| \leq 1} d z d \bar{z}|f(z)|^{2} e^{-2 \alpha(z, \bar{z})} .
$$

Once $f$ and $\alpha$ are given, the world-sheet embedding in target space follows from the solution of an auxiliary linear differential equation. Note that both the equation for $\alpha$ and the action depend only on the modulus of $f(z)$, so are invariant under $f(z) \rightarrow e^{i \varphi} f(z)$. The full string 
solution, and the boundary curve $X(\theta)$, may depend on $\varphi .^{4}$ This construction therefore leads to a one-parameter family of curves with the same strong coupling expectation value. We refer to $\varphi$ as the spectral parameter, and changing the curve induced by it as the spectral parameter deformation.

Let us provide further detail on how to extract the contour, given $f(z)$. As shown in [17], the behaviour of $\alpha(z, \bar{z})$ close to the boundary takes the form

$$
\alpha(z, \bar{z})=-\ln \xi+\xi^{2}(1+\xi) \beta_{2}(\theta)+\mathcal{O}\left(\xi^{4}\right), \quad \xi=1-r^{2},
$$

with some real function $\beta_{2}(\theta)$. One can then use (2.1) to fix the higher order terms in the expansion in terms of $\beta_{2}(\theta)$ and $f\left(e^{i \theta}\right)$. Examining the full string world-sheet near the boundary one can find an equation relating the Schwarzian derivative of the boundary contour $X(\theta)$ to the boundary values $\beta_{2}(\theta), f\left(e^{i \theta}\right)$ as well as $e^{i \varphi}$ :

$$
\begin{aligned}
\{X(\theta), \theta\} & \equiv \frac{X^{\prime \prime \prime}(\theta)}{X^{\prime}(\theta)}-\frac{3}{2}\left(\frac{X^{\prime \prime}(\theta)}{X^{\prime}(\theta)}\right)^{2}=-2 V(\theta), \\
V(\theta) & =-\frac{1}{4}+6 \beta_{2}(\theta)+2 i \operatorname{Im}\left(e^{2 i \theta} e^{i \varphi} f\left(e^{i \theta}\right)\right) .
\end{aligned}
$$

Note that (2.4) depends on the parametrization of $X(\theta)$ and it holds only for the parametrization arising in this construction, where $\theta$ is the polar angle in the world-sheet disk. If one starts instead with an arbitrarily parametrized curve $X(s(\theta))$, one would need to follow the procedure described in $[17,18]$ to find the "correct" parametrization related to the world-sheet description.

An alternative formulation of the problem is in terms of a Schrödinger problem. The requirement that the surface closes up smoothly inside the disk yields a consistency condition that fixes $\beta_{2}(\theta)$. One needs to tune $\beta_{2}(\theta)$ so that the solution of the Schrödinger equation

$$
-\chi^{\prime \prime}(\theta)+V(\theta) \chi(\theta)=0
$$

is anti-periodic in $\theta$ with period $2 \pi$ for any $\varphi$. We can also define the periodic function $\kappa(\theta) \equiv e^{-i \theta / 2} \chi(\theta)$, satisfying

$$
-\kappa^{\prime \prime}(\theta)-i \kappa^{\prime}(\theta)+\left(V(\theta)+\frac{1}{4}\right) \kappa(\theta)=0 .
$$

This alternative is purely algebraic and one has not to solve the cosh-Gordon equation. It does introduce a complication that to fully determine the solution at a fixed order in $\epsilon$ one has to include terms of higher orders, so it is not a very efficient algorithm.

Now we see why it is advantageous in this formalism to define the Wilson loop in terms of $f(z)$ rather than by the boundary contour $X(\theta)$ (assuming of course that we are interested in general contours, rather than a specific shape). First, a boundary contour $X(\theta)$ is not invariant under conformal transformations, which complicates a general classification of curves, whereas $f(z)$ is invariant. Second, the spectral parameter deformation is natural when starting with $f(z)$, and then solving for the boundary curve $X(\theta)$. The

\footnotetext{
${ }^{4}$ One can view $\varphi$ also as an integration constant arising in the solution of the auxiliary problem.
} 
inverse procedure, going from $X(\theta)$ to $f(z)$ and $\varphi$, requires also to reparametrize the curve in terms of the correct angle in the unit disc [17]. ${ }^{5}$ Lastly, turning on $\varphi$ by a spectral deformation, which modifies $f(z)$ by a constant phase, influences one part of the calculation outlined above.

\subsection{Perturbations around the circle}

Our starting point is the circle with $f(z)=0$. We label small deformations by $f(z)$ proportional to a small parameter $\epsilon$ and by their Taylor expansion around the origin as in (1.6). Clearly the circle is invariant under the spectral deformation $f(z) \rightarrow e^{i \varphi} f(z)$. The next simplest case is when $f(z)$ is a monomial, namely $f(z)=\epsilon a_{p} z^{p-2}$. The spectral deformation shifts the phase of $a_{p}$. The resulting generalized cosh-Gordon equation (2.1) is independent of $\theta$, which means $\alpha(r, \theta)=\alpha(r)$, and equation (2.3) implies that $\beta_{2}$ is a constant. Indeed we can absorb $\varphi$ in $z$, which amounts to a shift of $\theta$. Therefore, the spectral deformation is equivalent to rotations of the curve $X(\theta)$ and the VEV of the Wilson loop does not change. These choices of $f(z)$ were studied in [19] and the exact regularized area was found. The one-loop and two-loop expressions for such loops can be extracted from the expressions in subsequent sections.

We now turn to study deformations with general $f(z)$. The $\epsilon$-expansion of the Wilson loop's expectation value involves only even powers of $\epsilon$. Indeed flipping $f(z) \rightarrow-f(z)$ induces $X \rightarrow X^{*}$ in (2.4), which is a reflection in the $\left(x^{1}, x^{2}\right)$ plane. Note that the VEV of the Wilson loop at order $\epsilon^{2 l}$ is a degree $2 l$ polynomial in the Taylor coefficients $a_{p}$ and $\bar{a}_{p}$. So to this order it suffices to consider $f(z)$ which is a general polynomial with up to $2 l$ nonzero $a_{p}$.

The fact that there are no terms at order $\epsilon$ implies that expanding $X(\theta)$ to order $\epsilon$ contributes to the final answer only at order $\epsilon^{2}$. More generally, any perturbative calculation (like (3.1) and (3.11) below) vanishes when one $X\left(\theta_{i}\right)$ is a deformation of the circle at order $\epsilon^{2 l}$ and all other $X\left(\theta_{j}\right)$ are undeformed circles. We therefore require the expression for the curve $X\left(\theta_{i}\right)$ at order $\epsilon^{2 l-1}$.

We tackle the problem by expanding $X(\theta), \alpha(z, \bar{z})$ and $\beta_{2}(\theta)$ in power series in $\epsilon$, which allows to solve the nonlinear equation (2.1) and invert the Schwarzian derivative equation (2.4) perturbatively. The starting point is

$$
\begin{aligned}
\alpha(z, \bar{z}) & =\sum_{l=0}^{\infty} \epsilon^{2 l} \alpha^{(2 l)}(z, \bar{z}), & \alpha^{(0)}(z, \bar{z}) & =-\log \left(1-r^{2}\right), \\
\beta_{2}(\theta) & =\sum_{l=0}^{\infty} \epsilon^{2 l} \beta_{2}^{(2 l)}(\theta), & \beta_{2}^{(0)}(\theta) & =0
\end{aligned}
$$

where the lowest order terms match those for the circle. Plugging (2.7) and (1.6) into (2.1)

\footnotetext{
${ }^{5}$ An analytic solution for wavy circles was developed in [13] and a numerical approach for generic curves in [18], but both are complicated.
} 
gives differential recursion relations for $\alpha^{(2 l)}$. The first three are

$$
\begin{aligned}
\mathcal{L} \alpha^{(2)} & =4\left(1-r^{2}\right)^{2}|f / \epsilon|^{2}, \quad \mathcal{L}=\partial_{r}^{2}+\frac{\partial_{r}}{r}+\frac{\partial_{\theta}^{2}}{r^{2}}-\frac{8}{\left(1-r^{2}\right)^{2}}, \\
\mathcal{L} \alpha^{(4)} & =\frac{8\left(\alpha^{(2)}\right)^{2}}{\left(1-r^{2}\right)^{2}}-8\left(1-r^{2}\right)^{2}|f / \epsilon|^{2} \alpha^{(2)}, \\
\mathcal{L} \alpha^{(6)} & =\frac{16 \alpha^{(2)}\left(\left(\alpha^{(2)}\right)^{2}+3 \alpha^{(4)}\right)}{3\left(1-r^{2}\right)^{2}}-8\left(1-r^{2}\right)^{2}|f / \epsilon|^{2}\left(\alpha^{(4)}-\left(\alpha^{(2)}\right)^{2}\right) .
\end{aligned}
$$

To determine the VEV at order $\epsilon^{4}$, we only need to find $\alpha^{(2)}(z, \bar{z})$. It is useful to represent the holomorphic function as [13]

$$
f(z)=-\frac{1}{2}\left(z G^{\prime \prime \prime}(z)+3 G^{\prime \prime}(z)\right)
$$

where clearly

$$
G(z)=-2 \epsilon \sum_{p=2}^{\infty} \frac{a_{p} z^{p}}{p\left(p^{2}-1\right)} .
$$

The solution of the linear differential equation for $\alpha^{(2)}$ is then

$$
\begin{aligned}
\alpha^{(2)}(z, \bar{z})= & |W(z, \bar{z})|^{2}-\frac{z \bar{G}(\bar{z}) W(z, \bar{z})+\bar{z} G(z) \bar{W}(z, \bar{z})}{1-|z|^{2}} \\
& +\frac{1+|z|^{2}}{1-|z|^{2}}(T(z)+\bar{T}(\bar{z}))+z T^{\prime}(z)+\bar{z} \bar{T}^{\prime}(\bar{z}),
\end{aligned}
$$

where the first line is a particular solution to the inhomogeneous equation with

$$
W(z, \bar{z}) \equiv \frac{1}{2} z\left(|z|^{2}-1\right) G^{\prime \prime}(z)-G^{\prime}(z) .
$$

The second line is a general solution to the homogeneous equation, which is fixed by imposing the boundary condition that there are no poles for $\xi \rightarrow 0$ in (2.3). The result is

$$
T(z)=-2 \epsilon^{2} \sum_{p=2}^{\infty} \frac{\left|a_{p}\right|^{2}}{p^{2}\left(p^{2}-1\right)^{2}}-2 \epsilon^{2} \sum_{p=2}^{\infty} \sum_{q=p+1}^{\infty} \frac{(p+q) z^{q-p} a_{q} \bar{a}_{p}}{p\left(p^{2}-1\right) q\left(q^{2}-1\right)} .
$$

Equipped with $\alpha^{(2)}(z, \bar{z})$, we can read off $\beta_{2}(\theta)$ in two equivalent ways. Using (2.3) and expanding $\alpha^{(2)}$ to order $\xi^{2}$ gives

$$
\beta_{2}^{(2)}(\theta)=-\frac{1}{3} \sum_{p=2}^{\infty} \frac{\left|a_{p}\right|^{2}}{p^{2}-1} .
$$

In the alternative approach, based on the periodicity of $\kappa$, one can expand $\kappa(\theta)=$ $\sum_{l=0}^{\infty} \epsilon^{l} \kappa^{(l)}(\theta)$ with $\kappa^{(0)}(\theta)=1$ and the Schrödinger equation (2.6) becomes

$$
\begin{aligned}
& L_{1} \kappa^{(1)}=2 i \operatorname{Im}\left(e^{2 i \theta} e^{i \varphi} f\left(e^{i \theta}\right)\right), \quad L_{1} \kappa \equiv \kappa^{\prime \prime}+i \kappa^{\prime}, \\
& L_{1} \kappa^{(2)}=2 i \operatorname{Im}\left(e^{2 i \theta} e^{i \varphi} f\left(e^{i \theta}\right)\right) \kappa^{(1)}+6 \beta_{2}^{(2)}, \\
& L_{1} \kappa^{(3)}=2 i \operatorname{Im}\left(e^{2 i \theta} e^{i \varphi} f\left(e^{i \theta}\right)\right) \kappa^{(2)}+6 \beta_{2}^{(2)} \kappa^{(1)}, \\
& L_{1} \kappa^{(4)}=2 i \operatorname{Im}\left(e^{2 i \theta} e^{i \varphi} f\left(e^{i \theta}\right)\right) \kappa^{(3)}+6 \beta_{2}^{(2)} \kappa^{(2)}+6 \beta_{2}^{(4)},
\end{aligned}
$$


and so on. The inverse of $L_{1}$ on a basis of exponentials is given by

$$
L_{1}^{-1} e^{i k \theta}= \begin{cases}-\frac{e^{i k \theta}}{k(k+1)}, & k \neq 0,-1, \\ -i \theta, & k=0, \\ e^{-i \theta}(1+i \theta), & k=-1,\end{cases}
$$

and that allows to solve for $\kappa^{(l)}$. The kernel is $\operatorname{ker}\left(L_{1}\right)=c_{1}+c_{2} e^{-i \theta}$, so the modes 0 and -1 must not appear on the right-hand sides for the solution to be periodic. Indeed there are no such terms in $V$, so we can solve the first line. At higher orders, the requirement of periodicity of $\kappa^{(l)}$ imposes conditions on $\beta_{2}^{(l)}$, such that there are no $k=0,-1$ modes on the right-hand side. Unfortunately, this does not completely determine $\beta_{2}^{(l)}$, order by order, and constraints from the higher order terms are needed. Still, one can rederive (2.15) above from these requirements.

We can now solve perturbatively for the shape of the curve. We take

$$
X(\theta)=\exp \left(i \theta+\sum_{l=1}^{\infty} \epsilon^{l} g^{(l)}(\theta)\right)
$$

and plug it into the Schwarzian derivative equation (2.4). The first few equations are

$$
\begin{aligned}
& L_{2} g^{(1)}=4 \operatorname{Im}\left(e^{2 i \theta} e^{i \varphi} f\left(e^{i \theta}\right) / \epsilon\right), \quad L_{2} g \equiv g^{\prime \prime \prime}+g^{\prime}, \\
& L_{2} g^{(2)}=\frac{i}{2}\left(g^{(1) \prime}\right)^{2}-\frac{3 i}{2}\left(g^{(1) \prime \prime}\right)^{2}-i g^{(1) \prime} g^{(1) \prime \prime \prime}-12 i \beta_{2}^{(2)}, \\
& L_{2} g^{(3)}=g^{(1) \prime \prime \prime}\left(\left(g^{(1) \prime}\right)^{2}-i g^{(2) \prime}\right)-3 i g^{(1) \prime \prime} g^{(2) \prime \prime}+g^{(1) \prime}\left(3\left(g^{(1) \prime \prime}\right)^{2}+i g^{(2) \prime}-i g^{(2) \prime \prime \prime}\right) .
\end{aligned}
$$

Now we can use the inverse of $L_{2}$, which is

$$
L_{2}^{-1} e^{i k \theta}=\frac{i e^{i k \theta}}{k\left(k^{2}-1\right)}, \quad k \neq 0, \pm 1 .
$$

$L_{2}^{-1}$ acting on $e^{i k \theta}$ with $k=0, \pm 1$ would give non-periodic functions, but these powers never occur on the right-hand side.

Let us apply the above procedure. Plugging (1.6) into the first line of (2.19) gives

$$
L_{2} g^{(1)}=4 \operatorname{Im}\left(e^{2 i \theta} e^{i \varphi} f\left(e^{i \theta}\right)\right)=-2 i \epsilon \sum_{p=2}^{\infty}\left(a_{p} e^{i p \theta+i \varphi}-\bar{a}_{p} e^{-i p \theta-i \varphi}\right) .
$$

Inverting $L_{2}(2.20)$ gives $X(\theta)$ to linear order in $\epsilon$

$$
g^{(1)}(\theta)=2 \sum_{p=2}^{\infty} \frac{a_{p} e^{i(p \theta+\varphi)}+\bar{a}_{p} e^{-i(p \theta+\varphi)}}{p\left(p^{2}-1\right)} .
$$

The Fourier modes in (1.3) at this order follow from the comparison with (2.18):

$$
b_{n}= \begin{cases}0, & n=-1,0,1, \\ \frac{2 \epsilon a_{n} e^{i \varphi}}{n\left(n^{2}-1\right)}, & n \geq 2, \\ -\frac{2 \epsilon \bar{a}_{-n} e^{-i \varphi}}{n\left(n^{2}-1\right)}, & n \leq-2 .\end{cases}
$$


Let us record also the next term

$$
\begin{gathered}
g^{(2)}(\theta)=\sum_{p=2}^{\infty} \frac{p\left(5 p^{2}+1\right) a_{p}^{2} e^{2 i(p \theta+\varphi)}-4\left(4 p^{2}-1\right)\left|a_{p}\right|^{2}-p\left(5 p^{2}+1\right) \bar{a}_{p}^{2} e^{-2 i(p \theta+\varphi)}}{p^{2}\left(p^{2}-1\right)^{2}\left(4 p^{2}-1\right)} \\
+\sum_{p>q \geq 2}\left[\frac{4\left(p^{2}+3 p q+q^{2}+1\right)\left(a_{p} a_{q} e^{i((p+q) \theta+2 \varphi)}-\bar{a}_{p} \bar{a}_{q} e^{-i((p+q) \theta+2 \varphi)}\right)}{\left(p^{2}-1\right)\left(q^{2}-1\right)(p+q)\left((p+q)^{2}-1\right)}\right. \\
\left.-\frac{4 a_{p} \bar{a}_{q} e^{i(p-q) \theta}-4 \bar{a}_{p} a_{q} e^{-i(p-q) \theta}}{p\left(p^{2}-1\right)\left(q^{2}-1\right)}\right],
\end{gathered}
$$

from which we can similarly extract the $\epsilon^{2}$-correction to $b_{n}$. We have calculated also the general expression for $g^{(3)}(\theta)$, but have chosen not to write it, as it is very cumbersome and we explained in full detail the algorithm to evaluate it.

\section{Expectation value of perturbed circle}

We have two representations of the Wilson loop, in terms of the path as in (1.3) and in terms of the holomorphic function $f(z)$ on the string world-sheet. We now proceed to evaluate the expectation value of the Wilson loop at one and two loops at weak coupling in terms of the Fourier coefficients $b_{n}$ and, alternatively, in terms of the Taylor coefficients $a_{p}$. The map between these coefficients has been derived in the last section.

\subsection{One-loop order}

The one-loop expression for the Wilson loop is given by the double integral

$$
\langle W\rangle_{\lambda}=\frac{\lambda}{16 \pi^{2}} \oint d \theta_{1} d \theta_{2} I\left(\theta_{1}, \theta_{2}\right), \quad I\left(\theta_{1}, \theta_{2}\right)=-\frac{\operatorname{Re}\left(\dot{X}\left(\theta_{1}\right) \dot{\bar{X}}\left(\theta_{2}\right)\right)-\left|\dot{X}\left(\theta_{1}\right)\right|\left|\dot{X}\left(\theta_{2}\right)\right|}{\left|X\left(\theta_{1}\right)-X\left(\theta_{2}\right)\right|^{2}} .
$$

To quadratic order in $g(\theta)$, this is

$$
\begin{aligned}
I\left(\theta_{1}, \theta_{2}\right)= & \frac{1}{2}-\frac{i\left(e^{i \theta_{1}}+e^{i \theta_{2}}\right)\left(\dot{g}\left(\theta_{1}\right)-\dot{g}\left(\theta_{2}\right)\right)}{2\left(e^{i \theta_{1}}-e^{i \theta_{2}}\right)} \\
& +\frac{e^{i\left(\theta_{1}+\theta_{2}\right)}}{2\left(e^{i \theta_{1}}-e^{i \theta_{2}}\right)^{2}}\left[\left(g\left(\theta_{1}\right)-g\left(\theta_{2}\right)\right)^{2}-\dot{g}\left(\theta_{1}\right)^{2}-\dot{g}\left(\theta_{2}\right)^{2}+2 \cos \left(\theta_{1}-\theta_{2}\right) \dot{g}\left(\theta_{1}\right) \dot{g}\left(\theta_{2}\right)\right] .
\end{aligned}
$$

For the perturbed circle we plug in the Fourier representation of $g(\theta)(1.3)$ and expand to fixed order in powers of $b_{n}$, or alternatively $\epsilon$. We end up with a sum of many terms of the form

$$
\frac{e^{i n_{1} \theta_{1}+i n_{2} \theta_{2}}}{\left(e^{i \theta_{1}}-e^{i \theta_{2}}\right)^{p}},
$$

with varying $n_{1}, n_{2}$ and $p$. If we specify a finite set of Fourier modes and combine all the terms, the numerator factorizes and cancels the denominator. It is then trivial to do the integral, by picking out the zero-mode.

Unfortunately this factorization is not evident when the Fourier numbers are left as arbitrary variables $n_{i}$. There are two approaches to that case, first one can evaluate the 
result for many combinations of $n_{i}$ and try to fit it to a function of these integers. We chose instead a recursive algorithm to calculate the integrals

$$
A_{n_{1}, n_{2}}^{p}=\frac{1}{4 \pi^{2}} \oint d \theta_{1} d \theta_{2} \frac{e^{i n_{1} \theta_{1}+i n_{2} \theta_{2}}}{\left(e^{i \theta_{1}}-e^{i \theta_{2}}\right)^{p}}
$$

For $p=0$ this is simply $A_{n_{1}, n_{2}}^{0}=\delta_{0, n_{1}} \delta_{0, n_{2}}$. For $p>0$, instead of performing the integrals, we view them as formal objects satisfying the parity condition and recurrence relations, arising from combinations of integrands with factorizable numerators

$$
A_{n_{1}, n_{2}}^{p}=(-1)^{p} A_{n_{2}, n_{1}}^{p}, \quad \sum_{k=0}^{p}\left(\begin{array}{l}
p \\
k
\end{array}\right)(-1)^{k} A_{n_{1}+p-k, n_{2}+k}^{p}=A_{n_{1}, n_{2}}^{0}
$$

For $p=2$ this is solved by

$$
A_{n_{1}, n_{2}}^{2}=\frac{1}{4}\left|n_{1}-n_{2}\right| \delta_{2, n_{1}+n_{2}}+C_{n_{1}+n_{2}}^{(1)}
$$

with arbitrary $C_{n}^{(1)}$, which will cancel when we take the sum of integrals evaluating the Wilson loop. Note that each of the integrals (for $p>0$ ) is divergent, but the final expression for the Wilson loop at a given loop order is finite, so the arbitrary $C_{n}^{(1)}$ does in fact contain these divergences.

One can proceed to higher orders in the $g(\theta)$ expansion. At quartic order we find

$$
A_{n_{1}, n_{2}}^{4}=\frac{1}{96}\left|n_{1}-n_{2}\right|\left(\left(n_{1}-n_{2}\right)^{2}-4\right) \delta_{4, n_{1}+n_{2}}+C_{n_{1}+n_{2}}^{(2)}\left(n_{1}-n_{2}\right)^{2}+C_{n_{1}+n_{2}}^{(3)}
$$

where again $C^{(2)}$ and $C^{(3)}$ cancel out in the end result. In terms of the Fourier coefficients $b_{n}$, one finds to quartic order

$$
\begin{aligned}
\langle W\rangle_{\lambda}= & \frac{\lambda}{8}+\frac{\lambda}{4} \sum_{n=2}^{\infty} n\left(n^{2}-1\right)\left|b_{n}\right|^{2}+\frac{\lambda}{6} \sum_{n \geq m \geq l \geq 1}\left[3 S_{n, m, l}^{(1)} \times\right. \\
& \times l m n\left(2 l^{2}+2 m^{2}+2 n^{2}+3 l m+3 l n+3 m n-3\right)\left(b_{n} b_{m} b_{l} \bar{b}_{n+m+l}+\bar{b}_{n} \bar{b}_{m} \bar{b}_{l} b_{n+m+l}\right) \\
& +S_{n, m, l}^{(2)} l\left(l^{4}-l^{3} m-l^{3} n+l^{2} m^{2}-5 l^{2} m n+l^{2} n^{2}-6 m^{3} n-9 m^{2} n^{2}-6 m n^{3}+9 l m^{2} n\right. \\
& \left.\left.+9 l m n^{2}-4 l^{2}+l m+l n-m^{2}+8 m n-n^{2}+3\right)\left(b_{n} b_{m} \bar{b}_{l} \bar{b}_{n+m-l}+\bar{b}_{n} \bar{b}_{m} b_{l} b_{n+m-l}\right)\right],
\end{aligned}
$$

with the symmetry factors

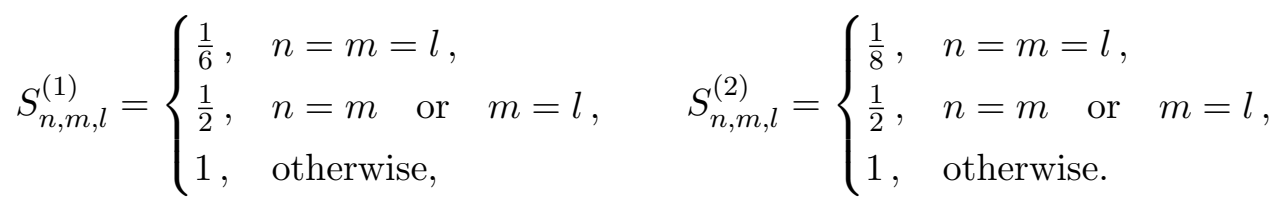


We can also express this in terms of the coefficients $a_{p}$ of the function $f(z)$. Plugging (2.22), (2.24) and the order $\epsilon^{3}$ term into the last equation, we find

$$
\begin{aligned}
\langle W\rangle_{\lambda}= & \frac{\lambda}{8}+\lambda \epsilon^{2} \sum_{p=2}^{\infty} \frac{\left|a_{p}\right|^{2}}{p\left(p^{2}-1\right)} \\
& +\frac{8}{3} \lambda \epsilon^{4} \sum_{p \geq q \geq r \geq 2} S_{p, q, r}^{(2)} \frac{a_{p} \bar{a}_{q} \bar{a}_{r} a_{q+r-p}+\bar{a}_{p} a_{q} a_{r} \bar{a}_{q+r-p}}{p\left(p^{2}-1\right) q\left(q^{2}-1\right) r\left(r^{2}-1\right)(q+r)\left((q+r)^{2}-1\right)} \\
& \times\left[4 p^{2}(q+r)\left((q+r)^{2}-1\right)+p\left(-q^{4}+2 q^{3} r+12 q^{2} r^{2}+2 q r^{3}-r^{4}+q^{2}+8 q r+r^{2}\right)\right. \\
& \left.-(q+r)\left(2 q^{4}+3 q^{3} r-4 q^{2} r^{2}+3 q r^{3}+2 r^{4}+q^{2}-5 q r+r^{2}-3\right)\right]+\mathcal{O}\left(\epsilon^{6}\right),
\end{aligned}
$$

with the same symmetry factor as above. It is easy to see that the order $\epsilon^{2}$ term is an alternative representation of the coefficient of the Bremsstrahlung function. At order $\epsilon^{4}$ one could have had in principle any polynomial of degree 4 in $a_{p}$ and $\bar{a}_{p}$ with up to four different $p$ 's, but most of those vanish. Instead, we find that they are all of the form $a_{p_{1}} a_{p_{2}} \bar{a}_{p_{3}} \bar{a}_{p_{4}}$, with $p_{1}+p_{2}-p_{3}-p_{4}=0$. Note that all the $\varphi$ dependence in (2.24) completely cancels in this expression and it is $\varphi$ independent, confirming the observation in [13]! As we discuss in section 4 , this independence is generally violated at order $\epsilon^{6}$ and above.

\section{$3.2 \quad$ Two-loop order}

A similar analysis can also be done at two-loops order by virtue of a compact formula [16] which incorporates all two-loop Feynman diagrams for a curve in $\mathbb{R}^{2}$

$$
\begin{aligned}
\langle W\rangle_{\lambda^{2}}= & \frac{\lambda^{2}}{128 \pi^{4}} \oint d \theta_{1} d \theta_{2} d \theta_{3}\left[\varepsilon\left(\theta_{1}, \theta_{2}, \theta_{3}\right) I\left(\theta_{1}, \theta_{3}\right) \frac{\operatorname{Re}\left(\left(X\left(\theta_{3}\right)-X\left(\theta_{2}\right)\right) \dot{\bar{X}}\left(\theta_{2}\right)\right)}{\left|X\left(\theta_{3}\right)-X\left(\theta_{2}\right)\right|^{2}}\right. \\
& \left.\times \log \frac{\left|X\left(\theta_{1}\right)-X\left(\theta_{2}\right)\right|^{2}}{\left|X\left(\theta_{3}\right)-X\left(\theta_{1}\right)\right|^{2}}\right]+\frac{\lambda^{2}}{2}\left(\frac{1}{16 \pi^{2}} \oint d \theta_{1} d \theta_{2} I\left(\theta_{1}, \theta_{2}\right)\right)^{2} \\
& -\frac{\lambda^{2}}{64 \pi^{4}} \int_{\theta_{1}>\theta_{2}>\theta_{3}>\theta_{4}} d \theta_{1} d \theta_{2} d \theta_{3} d \theta_{4} I\left(\theta_{1}, \theta_{3}\right) I\left(\theta_{2}, \theta_{4}\right),
\end{aligned}
$$

with $I\left(\theta_{1}, \theta_{2}\right)$ from (3.1). The first integral is due to cubic interactions and $\varepsilon$ is completely antisymmetric in permutations of its arguments and takes the value 1 for $\theta_{1}>\theta_{2}>\theta_{3}$.

The procedure we employ to evaluate these integrals is a generalization of the one-loop case, but it is significantly more complicated and is explained in appendix A. Expanding (3.11) in powers of the Fourier coefficients $b_{n}$ gives integrals with all the $X(\theta)$ factors in (3.11) replaced by exponentials (and arbitrary powers of differences of exponentials in the denominator), as in (A.1) and (A.8). If we consider the cases of a trivial denominator, the integrals are convergent, and these serve again as the boundary conditions for recursion relations for the integrals with arbitrary denominator, (A.5) and (A.10). As in the oneloop integrals, there are ambiguities in solving the recursion relations which are solutions to homogeneous equations, but they drop out from the final expressions. The complexity of the recursion relations prevented us from finding closed-form solutions like (3.6) and (3.7), but for any finite set of Fourier modes with mode numbers $n_{i}$, we can solve the recursion with the aid of a computer. 
At order $\epsilon^{2}$ we know that the result should be determined by the Bremsstrahlung function, and in particular should be proportional to the order $\epsilon^{2}$ term in (3.10). To verify this, we considered

$$
f(z)=\epsilon\left(a_{p} z^{p-2}+a_{q} z^{q-2}\right), \quad \text { with } \quad 2 \leq q<p \leq 12 .
$$

We used the algorithm in appendix A to find the result

$$
\langle W\rangle_{\lambda^{2}}=\frac{\lambda^{2}}{192}+\frac{\lambda^{2} \epsilon^{2}}{12} \sum_{p=2}^{\infty} \frac{\left|a_{p}\right|^{2}}{p\left(p^{2}-1\right)}+\mathcal{O}\left(\epsilon^{4}\right) .
$$

The functional form and numerical prefactor indeed agree with the two-loop order of (1.5).

At order $\epsilon^{4}$ the result should be quartic in $a_{p_{i}}$ and $\bar{a}_{p_{i}}$, so we need consider $f(z)$ with at most four terms

$$
f(z)=\epsilon\left(a_{p_{1}} z^{p_{1}-2}+a_{p_{2}} z^{p_{2}-2}+a_{p_{3}} z^{p_{3}-2}+a_{p_{4}} z^{p_{4}-2}\right) .
$$

We computed the result for all such $f(z)$ of degree no larger than 10

$$
2 \leq p_{4}<p_{3}<p_{2}<p_{1} \leq 12 .
$$

We find that of all possible degree-4 monomials in $a_{p_{i}}$ and $\bar{a}_{p_{i}}$, the only non-zero contributions come from terms of the form $a_{p} a_{q} \bar{a}_{r} \bar{a}_{p+q-r}$, with arbitrary $p, q, r$. This fits exactly the pattern at one-loop order (3.10).

The fact that the allowed monomials contain an equal number of $a$ and $\bar{a}$ immediately implies that there is no spectral parameter dependence in the final expression. We conjecture that these properties extend beyond this range of $p_{i}$.

Unlike (3.10), the numerical coefficients of these monomials are not finite degree rational functions of the $p_{i}$. Indeed, already the simplest triple integrals in (A.4) includes harmonic numbers, so we expect the coefficients to be expressible in terms of harmonic numbers, but we could not guess a simple form.

Another difference from (3.10) is that these coefficients are actually not rational, they include both rational pieces and $\pi^{-2}$ times rational numbers. It is easy to see from the expressions in appendix A, that the triple integral in (3.11) (with its prefactor) is proportional to $\pi^{-2}$, the square of the one-loop order is clearly rational, and the quadruple integral includes both rational and $\pi^{-2}$ pieces. In fact, the $\pi^{0}$ part of the quadruple integral is easy to trace, and is related in a simple way to the one-loop integral, as follows.

The integrand of the quadruple integral is the same as the square of the one-loop integrand, and as mentioned above equation (3.4), once one chooses a particular set of Fourier modes, the numerator factorizes to cancel the denominator, leading to the integral (A.13). The resulting expression there seems rational, and further divided by $64 \pi^{4}$, but positive powers of $\pi$ appear from degenerations in the coefficients $n_{i}$ (and it vanishes without those degenerations). A factor of $\pi^{4}$ comes only from full degeneration $n_{i} \rightarrow 0$, giving the measure factor $\pi^{4} / 24$ (which is divided by $64 \pi^{4}$ ). Since the one-loop answer is solely from the zero-mode in the double integral and the integrands are the same, we find that the $\pi^{0}$ part of the last line in (3.11) is equal to $-\left(\langle W\rangle_{\lambda}\right)^{2} / 6$. 
Combining with the square of the one-loop integral on the second line of (3.11), we get the very simple expression for the rational part of the two loop integral

$$
\left.\langle W\rangle_{\lambda^{2}}\right|_{\pi^{0}}=\frac{1}{3}\left(\langle W\rangle_{\lambda}\right)^{2} .
$$

Note that this statement is true to all orders in $\epsilon$, so the difficulty is in calculating the $\pi^{-2}$ part in the two-loop VEV. Clearly the $\epsilon^{2}$ part in (3.13), which is just the two-loop Bremsstrahlung function, is rational and is given by this recursion. Likewise, we expect at $k$-th loop order to find terms proportional to $\pi^{0}, \cdots, \pi^{2-2 k}$, and all but the last term would be related to iterations of the lower loop expressions.

\section{Spectral dependence at higher orders in $\epsilon$}

In the cases studied in [13] there was spectral dependence starting from order $\epsilon^{8}$. As shown in the previous section, there is no dependence at order $\epsilon^{4}$, so here we address the possible spectral dependence at order $\epsilon^{6}$ and further patterns of spectral dependence at higher orders.

It should be possible to repeat the full analysis in the previous section for order $\epsilon^{6}$ with a bit more computing power, but we chose to do a partial survey by considering binomial $f(z)$, as in (3.12). Examining the cases with $2 \leq q<p \leq 22$ at one-loop order, we found that there was indeed spectral dependence in some examples at order $\epsilon^{6}$. The only such dependence is of the form $e^{ \pm 2 i \varphi}$ and happens only for $p=2 q$ or $p=3 q$. Specifically, the answer is a linear combination of terms of the form

$$
\begin{array}{lll}
\left|a_{q}\right|^{6}, & \left|a_{p}\right|^{2}\left|a_{q}\right|^{4}, & \left|a_{p}\right|^{4}\left|a_{q}\right|^{2}, \\
\bar{a}_{2 q}^{2} a_{q}^{4} e^{2 i \varphi}, & \bar{a}_{3 q}\left|a_{3 q}\right|^{2} a_{q}^{3} e^{2 i \varphi}, & \bar{a}_{3 q} a_{q}^{3}\left|a_{q}\right|^{2} e^{2 i \varphi}, \\
a_{2 q}^{2} \bar{a}_{q}^{4} e^{-2 i \varphi}, & a_{3 q}\left|a_{3 q}\right|^{2} \bar{a}_{q}^{3} e^{-2 i \varphi}, & a_{3 q} \bar{a}_{q}^{3}\left|a_{q}\right|^{2} e^{-2 i \varphi} .
\end{array}
$$

At one-loop the coefficients are rational functions of $q$ and $p$, a bit more complicated than in (3.10), and at two-loops (where we did a far less extensive survey) they are harmonic numbers.

It is obvious that $e^{2 i \varphi}$ would appear in terms where the number of $a$ 's is two more than $\bar{a}$. But we see that there are no terms with $e^{4 i \varphi}$, which would require five $a$ 's and one $\bar{a}$. Furthermore we see that in all cases the total sum of $p$ and $q$ (weighted by degeneracy and a negative sign for $\bar{a}$ ) vanishes. We find then a pattern that generalizes the result at order $\epsilon^{4}$ and could hold for general order. At order $\epsilon^{2 l}$ we therefore expect only terms of the form

$$
a_{p_{1}} \cdots a_{p_{j}} \bar{a}_{q_{1}} \cdots \bar{a}_{q_{2 l-j}} e^{2(j-l) i \varphi}
$$

with the constraints

$$
2 \leq j \leq 2 l-2, \quad \sum_{i=1}^{j} p_{i}-\sum_{i=1}^{2 l-j} q_{i}=0 .
$$

Clearly for $l=2$, one has $j=2$ and no spectral dependence. For $l=3$ there are many solutions, but if we restrict to binomials, so $p_{i}$ and $q_{i}$ can take only two values, we find indeed only the solutions in (4.1). 
In the binomial examples we checked beyond this order and at order $\epsilon^{8}$, we have found terms consistent with, among others,

$$
\bar{a}_{3 q}^{2} a_{q}^{6} e^{4 i \varphi}, \quad \bar{a}_{5 q} a_{q}^{5}\left|a_{q}\right|^{2} e^{4 i \varphi}, \quad \bar{a}_{5 q}^{3} a_{3 q}^{5} e^{2 i \varphi} .
$$

\section{$5 \quad$ Strong coupling}

One can also study perturbations of the circular Wilson loop at strong coupling, where it is described by a classical string in $A d S_{3}$. The VEV of the Wilson loop is given by the regularized area of the string, which is expressed in terms of $f(z)$ and $\alpha(z, \bar{z})$ in $(2.2)$. As mentioned before, this expression is independent of the spectral parameter $\varphi$ to any order in $\epsilon$, but given the expressions above, we can plug in the expressions for $f(z)$ in (1.6) and $\alpha^{(2)}(z, \bar{z})$ in $(2.12)$ to find the VEV in terms of $a_{p}$ 's.

This was done for a particular deformation of the circle in [19], based on the perturbative algorithm of [13] reviewed above. Here this is repeated for arbitrary $a_{p}$ to quartic order in $\epsilon$, giving

$$
\begin{aligned}
& A_{\text {reg }}=-2 \pi \sqrt{\lambda}-8 \pi \sqrt{\lambda} \epsilon^{2} \sum_{p=2}^{\infty} \frac{\left|a_{p}\right|^{2}}{p\left(p^{2}-1\right)} \\
&-64 \pi \sqrt{\lambda} \epsilon^{4} \sum_{p \geq q \geq r \geq 2} S_{p, q, r}^{(2)} \frac{a_{p} \bar{a}_{q} \bar{a}_{r} a_{q+r-p}+\bar{a}_{p} a_{q} a_{r} \bar{a}_{q+r-p}}{p\left(p^{2}-1\right) q\left(q^{2}-1\right) r\left(r^{2}-1\right)(q+r)\left((q+r)^{2}-1\right)} \\
& \times\left[p^{2}(q+r)\left((q+r)^{2}-1\right)+p q r\left(q^{2}+3 q r+r^{2}+1\right)\right. \\
&\left.\quad-(q+r)\left(q^{4}+q^{3} r-q^{2} r^{2}+q r^{3}+r^{4}-q^{2}-q r-r^{2}\right)\right],
\end{aligned}
$$

with $S_{p, q, r}^{(2)}$ defined in (3.9). Note the similarities to (3.10), with the same denominators and the only difference being the polynomial in $p, q$ and $r$ in the numerator. In both expressions this is a degree 5 polynomial symmetric in $(q, r)$ with $p$ of at most degree 2 .

\section{Discussion}

In this paper we undertook to analyze the expectation values of deformed circular Wilson loops in $\mathcal{N}=4 \mathrm{SYM}$ to quartic order in the deformation parameter. We presented explicit expressions for the expectation values at one-loop at weak coupling and at leading order at strong coupling. At two-loop order we provided all details of an explicit algorithm that calculates the VEV for particular deformations very efficiently.

As the stated goal of this project we have confirmed that the expectation value is independent of the spectral parameter at this order in the deformation and the perturbative orders that we examined. We expect this to hold to all orders in perturbation theory.

At higher orders in the deformation $\left(\epsilon^{6}\right.$ and above), we found that generically there is spectral dependence in the expectation value of the Wilson loop, so different Wilson loop that are related to each other via this deformation, an exact strong-coupling symmetry, do not share a VEV at weak coupling. 
Still, the pattern of spectral dependence as expressed in equation (4.2), which is the pattern we conjecture to hold to any order in the deformation, is exceedingly restrictive and simple. It is a striking result of our examinations that when representing the Wilson loop in terms of the sigma-model (Pohlmeyer) representation, i.e., in terms of $e^{i \varphi} f(z)$, there is such a simple pattern of allowed terms in the expression for the VEV of the Wilson loop at weak coupling. Furthermore, the one-loop expressions at order $\epsilon^{4}$ and at strong coupling share a very similar functional form as rational functions of the orders of terms in the Taylor expansion of $f(z)$.

It is very natural that the result of integration requires the sum of the Fourier coefficients to vanish. This precludes direct interaction between one very high Fourier mode and several low ones, a natural structure limiting UV-IR mixing. It is more surprising that similar structures arise, at weak coupling, in the calculation in terms of the Taylor coefficients of $f(z)$. Their weighted sum also seems to vanish and the spectral parameter can appear only in very specific combinations. This again can be thought of as avoiding UV-IR mixing, though it is surprising that it survives from the string description to weak coupling.

The spectral independence at order $\epsilon^{4}$ and other structures are hints to hidden symmetries in the expectation values of Wilson loop operators, which presumably have to do with all-loop integrability. A formalism exists for the all-loop integrability of the anomaly of cusps in the Wilson loop and for dimensions of insertions into the Wilson loop, but not for the question at hand [20-24], related to smooth Wilson loops. Perhaps there is a way of recombining results for 3-point (and higher) correlators of insertions into the Wilson loop that would prove our conjectures [8, 9, 25-29]. It would also be very interesting to understand any possible connections to other discussions of all-loop Yangian symmetry of smooth Wilson loops, as in $[14,15]$ as well as the TBA formulation of the problem in [30].

An obvious generalization of our calculation is to study Wilson loops not restricted to $\mathbb{R}^{2}$. This can be done, since it is understood how to solve for the boundary curve for open strings in the full $A d S_{5}$ [31]. Though there is no explicit solution of the string sigmamodel, as in the $A d S_{3}$ case, there is enough information to describe the boundary curve from details of a classical integrable model.

The same $A d S_{3}$ sigma-model describes also Wilson loops in ABJM theory restricted to a plane, so the strong coupling analysis applies directly there as well (with the appropriate redefinition of the coupling). This should capture the strong coupling limit of both locally $1 / 6$ BPS [32-34] and 1/2 BPS [35] Wilson loops of this theory. It should be possible to repeat our two-loop analysis for these loops and see what is the spectral dependence in the two classes of loops and whether they are related to each other.

It may be possible to extend our strong coupling analysis beyond the leading order and include the first string fluctuations, as was done for cusps in [36-38] and for circular Wilson loops in [39-44]. It would be very interesting to see whether spectral dependence (at order $\epsilon^{6}$ ) appears in that calculation. 


\section{Acknowledgments}

We acknowledge useful discussions with Bartomeu Fiol, Martín Kruczenski, Georgios Papathanasiou and Kostya Zarembo. The work of A.D. was supported by the Swedish Research Council (VR) grant 2013-4329. The research of N.D. was supported in part by the Science \& Technology Facilities Council via the consolidated grant number ST/P000258/1 and by a joint KCL-FAPESP fellowship. He is grateful to the hospitality of the University of São Paulo, Peking University, Weizmann Institute, Nordita and IIP Natal during the course of this work. D.T. acknowledges partial financial support from CNPq, the FAPESP grants 2014/18634-9 and 2015/17885-0, the joint KCL-FAPESP grant 2017/50435-4, and the FAPESP grant 2016/01343-7 through ICTP-SAIFR. The work of E.V. is funded by the FAPESP grants 2014/18634-9 and 2016/09266-1, and by the STFC grant ST/P000762/1. He thanks Yunfeng Jiang and ETH Zurich for hospitality while this project was in preparation.

\section{A Two-loop integrals}

In this appendix we present the details of the two-loop calculation, which amounts to finding the Fourier representation of (3.11). Expanding the integrands in powers of $g(\theta)$ (see (1.3)) gives several types of basic integrals studied below.

\section{A.1 Logarithmic integrals}

The first set of integrals encountered in the expansion of (3.11) in powers of $b_{n_{i}}$ are

$$
B_{n_{1}, n_{2}, n_{3}}^{p_{1}, p_{2}, p_{3}}=-\frac{i}{8 \pi^{2}} \oint d \theta_{1} d \theta_{2} d \theta_{3} \varepsilon\left(\theta_{1}, \theta_{2}, \theta_{3}\right) \frac{e^{i\left(n_{1} \theta_{1}+n_{2} \theta_{2}+n_{3} \theta_{3}\right)} \log \left(2-2 \cos \left(\theta_{1}-\theta_{2}\right)\right)}{\left(e^{i \theta_{1}}-e^{i \theta_{2}}\right)^{p_{1}}\left(e^{i \theta_{2}}-e^{i \theta_{3}}\right)^{p_{2}}\left(e^{i \theta_{3}}-e^{i \theta_{1}}\right)^{p_{3}}} .
$$

For vanishing $p$ 's, the integrals $B_{n_{1}, n_{2}, n_{3}}^{0,0,0}$ are quite simple. We perform the calculation for real $n_{i}$ and take the limit to the integers at the end.

Expanding the antisymmetric symbol and integrating over $\theta_{3}$ gives

$$
\begin{aligned}
& \frac{1}{8 \pi^{2} n_{3}} \int_{0}^{2 \pi} d \theta_{1} \int_{0}^{\theta_{1}} d \theta_{2}\left(e^{i\left(n_{1} \theta_{1}+n_{2} \theta_{2}\right)}-e^{i\left(n_{2} \theta_{1}+n_{1} \theta_{2}\right)}\right)\left(2 e^{i n_{3} \theta_{1}}-2 e^{i n_{3} \theta_{2}}+1-e^{2 \pi i n_{3}}\right) \\
& \quad \times \log \left(2-2 \cos \left(\theta_{1}-\theta_{2}\right)\right) .
\end{aligned}
$$

The change of variables $\theta_{1}=(u-v) / 2, \theta_{2}=(u+v) / 2$ allows to integrate over $u$, resulting in

$$
\begin{aligned}
-\frac{i}{8 \pi^{2}} \int_{-2 \pi}^{0} d v( & \frac{2\left(e^{i n_{3} v}-1\right)\left(e^{2 \pi i\left(n_{1}+n_{2}+n_{3}\right)}-e^{-i\left(n_{1}+n_{2}+n_{3}\right) v}\right)}{n_{1}+n_{2}+n_{3}} \\
& \left.+\frac{\left(e^{2 \pi i n_{3}}-1\right)\left(e^{2 \pi i\left(n_{1}+n_{2}\right)}-e^{-i\left(n_{1}+n_{2}\right) v}\right)}{n_{1}+n_{2}}\right) \frac{e^{i n_{1} v}-e^{i n_{2} v}}{n_{3}} \log (2-2 \cos v) .
\end{aligned}
$$


Now we can take the $n_{i}$ to be integer, noting that no poles arise even when the sums of $n_{i}$ in the denominators vanish. The result of the $v$ integration is then

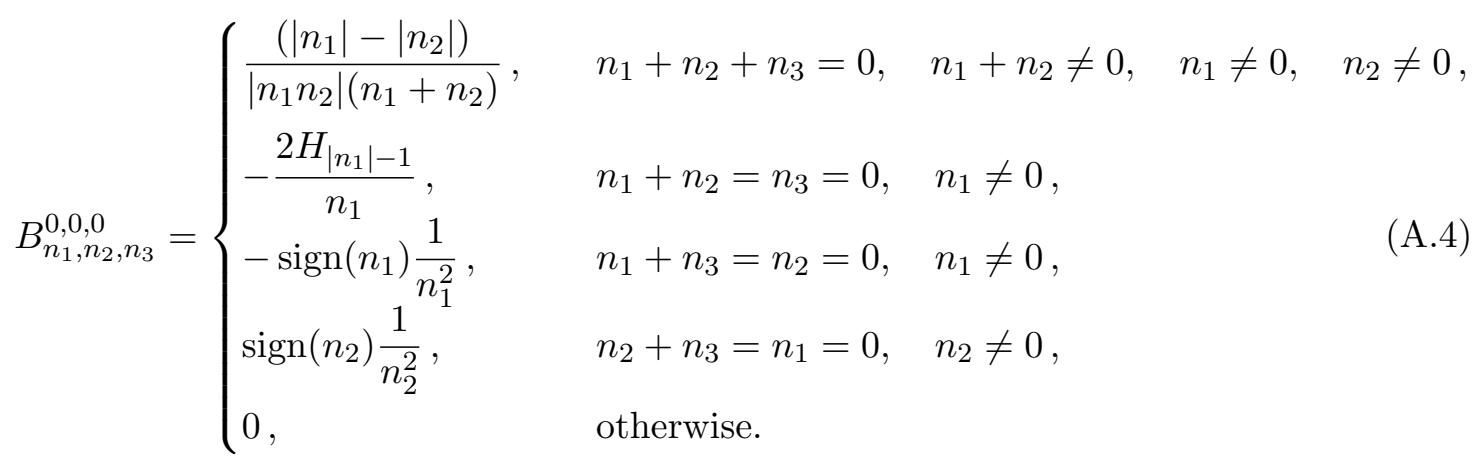

Here $H_{n}=\sum_{m=1}^{\infty} 1 / m$ are the harmonic numbers.

For positive $p_{1}, p_{2}, p_{3}$, we do not evaluate $B_{n_{1}, n_{2}, n_{3}}^{p_{1}, p_{2}, p_{3}}$ by integration, but rather impose the consistency and recursion relations

$$
\begin{gathered}
B_{n_{1}, n_{2}, n_{3}}^{p_{1}, p_{2}, p_{3}}=(-1)^{p_{1}+p_{2}+p_{3}+1} B_{n_{2}, n_{1}, n_{3}}^{p_{1}, p_{3}, p_{2}}, \\
\sum_{k_{1}=0}^{p_{1}} \sum_{k_{2}=0}^{p_{2}} \sum_{k_{3}=0}^{p_{3}}\left(\begin{array}{l}
p_{1} \\
k_{1}
\end{array}\right)\left(\begin{array}{l}
p_{2} \\
k_{2}
\end{array}\right)\left(\begin{array}{l}
p_{3} \\
k_{3}
\end{array}\right)(-1)^{k_{1}+k_{2}+k_{3}} B_{n_{1}+p_{1}+k_{3}-k_{1}, n_{2}+p_{2}+k_{1}-k_{2}, n_{3}+p_{3}+k_{2}-k_{3}}^{p_{1}, p_{3}}=B_{n_{1}, n_{2}, n_{3}}^{0,0,0} .
\end{gathered}
$$

The coefficients appearing in the calculation of the Wilson loop to quadratic order in $b_{n_{i}}$ are $B_{n_{1}, n_{2}, n_{3}}^{1,2,2}$. Restricting by parity symmetry to $n_{1} \leq n_{2}$ we find that:

- For $n_{1} \leq n_{2} \leq n_{1}+3$ one can choose the coefficients $B_{n_{1}, n_{2}, n_{3}}^{1,2,2}$ freely, and we set them to zero for simplicity.

- The coefficients with $n_{2}>n_{1}+3$ are generated recursively through equation (A.5) (with the replacements $n_{2} \rightarrow n_{2}-3, n_{3} \rightarrow n_{3}-2$ )

$$
\begin{aligned}
B_{n_{1}, n_{2}, n_{3}}^{1,2,2}= & -B_{n_{1}, n_{2}-3, n_{3}-2}^{0,0,0}-B_{n_{1}, n_{2}-2, n_{3}+2}^{1,2,2}+2 B_{n_{1}, n_{2}-1, n_{3}+1}^{1,2,2}+B_{n_{1}+1, n_{2}-3, n_{3}+2}^{1,2,2} \\
& -3 B_{n_{1}+1, n_{2}-1, n_{3}}^{1,2,2}+2 B_{n_{1}+1, n_{2}, n_{3}-1}^{1,2,2}-2 B_{n_{1}+2, n_{2}-3, n_{3}+1}^{1,2,}+3 B_{n_{1}+2, n_{2}-2, n_{3}}^{1,2,2} \\
& -B_{n_{1}+2, n_{2}, n_{3}-2}^{1,2,}+B_{n_{1}+3, n_{2}-3, n_{3}}^{1,2,2}-2 B_{n_{1}+3, n_{2}-2, n_{3}-1}^{1,2,2}+B_{n_{1}+3, n_{2}-1, n_{3}-2}^{1,2,2}
\end{aligned}
$$

At quartic order in the $b_{n_{i}}$ we need the coefficients $B_{n_{1}, n_{2}, n_{3}}^{3,4}$. Taking, without loss in generality, $n_{1} \leq n_{2}$ we have

- The coefficients with $n_{1} \leq n_{2} \leq n_{1}+7$ can be chosen freely and we set them to zero. 
- For $n_{1}+7<n_{2}$ the coefficients are generated recursively from (A.5):

$$
\begin{aligned}
& B_{n_{1}, n_{2}, n_{3}}^{3,4,4}=-B_{n_{1}, n_{2}-7, n_{3}-4}^{0,0,0}-B_{n_{1}, n_{2}-4, n_{3}+4}^{3,4}+4 B_{n_{1}, n_{2}-3, n_{3}+3}^{3,4,4}-6 B_{n_{1}, n_{2}-2, n_{3}+2}^{3,4,4} \\
& +4 B_{n_{1}, n_{2}-1, n_{3}+1}^{3,4,4}+3 B_{n_{1}+1, n_{2}-5, n_{3}+4}^{3,4,4}-8 B_{n_{1}+1, n_{2}-4, n_{3}+3}^{3,4,4}+2 B_{n_{1}+1, n_{2}-3, n_{3}+2}^{3,4,4} \\
& +12 B_{n_{1}+1, n_{2}-2, n_{3}+1}^{3,4}-13 B_{n_{1}+1, n_{2}-1, n_{3}}^{3,4,4}+4 B_{n_{1}+1, n_{2}, n_{3}-1}^{3,4,4}-3 B_{n_{1}+2, n_{2}-6, n_{3}+4}^{3,4,4} \\
& +24 B_{n_{1}+2, n_{2}-4, n_{3}+2}^{3,4}-36 B_{n_{1}+2, n_{2}-3, n_{3}+1}^{3,4,4}+9 B_{n_{1}+2, n_{2}-2, n_{3}}^{3,4,4}+12 B_{n_{1}+2, n_{2}-1, n_{3}-1}^{3,4,4} \\
& -6 B_{n_{1}+2, n_{2}, n_{3}-2}^{3,4}+B_{n_{1}+3, n_{2}-7, n_{3}+4}^{3,4,}+8 B_{n_{1}+3, n_{2}-6, n_{3}+3}^{3,4,4}-24 B_{n_{1}+3, n_{2}-5, n_{3}+2}^{3,4,4} \\
& +45 B_{n_{1}+3, n_{2}-3, n_{3}}^{3,4,4}-36 B_{n_{1}+3, n_{2}-2, n_{3}-1}^{3,4,4}+2 B_{n_{1}+3, n_{2}-1, n_{3}-2}^{3,4,4}+4 B_{n_{1}+3, n_{2}, n_{3}-3}^{3,4,4} \\
& -4 B_{n_{1}+4, n_{2}-7, n_{3}+3}^{3,4}-2 B_{n_{1}+4, n_{2}-6, n_{3}+2}^{3,4,4}+36 B_{n_{1}+4, n_{2}-5, n_{3}+1}^{3,4,4}-45 B_{n_{1}+4, n_{2}-4, n_{3}}^{3,4,4} \\
& +24 B_{n_{1}+4, n_{2}-2, n_{3}-2}^{3,4,}-8 B_{n_{1}+4, n_{2}-1, n_{3}-3}^{3,4,4}-B_{n_{1}+4, n_{2}, n_{3}-4}^{3,4,4}+6 B_{n_{1}+5, n_{2}-7, n_{3}+2}^{3,4,4} \\
& -12 B_{n_{1}+5, n_{2}-6, n_{3}+1}^{3,4,}-9 B_{n_{1}+5, n_{2}-5, n_{3}}^{3,4,4}+36 B_{n_{1}+5, n_{2}-4, n_{3}-1}^{3,4,4}-24 B_{n_{1}+5, n_{2}-3, n_{3}-2}^{3,4,4} \\
& +3 B_{n_{1}+5, n_{2}-1, n_{3}-4}^{3,4,4}-4 B_{n_{1}+6, n_{2}-7, n_{3}+1}^{3,4,4}+13 B_{n_{1}+6, n_{2}-6, n_{3}}^{3,4,4}-12 B_{n_{1}+6, n_{2}-5, n_{3}-1}^{3,4,4} \\
& -2 B_{n_{1}+6, n_{2}-4, n_{3}-2}^{3,4,4}+8 B_{n_{1}+6, n_{2}-3, n_{3}-3}^{3,4,4}-3 B_{n_{1}+6, n_{2}-2, n_{3}-4}^{3,4,4}+B_{n_{1}+7, n_{2}-7, n_{3}}^{3,4,4} \\
& -4 B_{n_{1}+7, n_{2}-6, n_{3}-1}^{3,4,4}+6 B_{n_{1}+7, n_{2}-5, n_{3}-2}^{3,4,}-4 B_{n_{1}+7, n_{2}-4, n_{3}-3}^{3,4}+B_{n_{1}+7, n_{2}-3, n_{3}-4}^{3,4,4} .
\end{aligned}
$$

\section{A.2 Non-logarithmic part in two-loop interacting diagrams}

Another class of integrals arising from the expansion of the first term in (3.11) in powers of the Fourier coefficients $b_{n_{i}}$ are

$$
C_{n_{1}, n_{2}, n_{3}}^{p_{1}, p_{2}, p_{3}}=-\frac{i}{8 \pi^{2}} \oint d \theta_{1} d \theta_{2} d \theta_{3} \varepsilon\left(\theta_{1}, \theta_{2}, \theta_{3}\right) \frac{e^{i n_{1} \theta_{1}+i n_{2} \theta_{2}+i n_{3} \theta_{3}}}{\left(e^{i \theta_{1}}-e^{i \theta_{2}}\right)^{p_{1}}\left(e^{i \theta_{2}}-e^{i \theta_{3}}\right)^{p_{2}}\left(e^{i \theta_{3}}-e^{i \theta_{1}}\right)^{p_{3}}} .
$$

The integral with all $p$ 's vanishing is finite and evaluates to

$$
C_{n_{1}, n_{2}, n_{3}}^{0,0,0}=\left\{\begin{array}{lll}
\frac{1}{n_{2}} & n_{2}+n_{3}=n_{1}=0, & n_{2} \neq 0 \\
\frac{1}{n_{3}} & n_{1}+n_{3}=n_{2}=0, & n_{3} \neq 0 \\
\frac{1}{n_{1}} & n_{1}+n_{2}=n_{3}=0, & n_{1} \neq 0 \\
0 & \text { otherwise. }
\end{array}\right.
$$

The other integrals are divergent, but as explained before, we expect the divergences to cancel in the final expressions and we choose a "regularization" of them by requiring the symmetry and recursion relations

$$
\begin{gathered}
C_{n_{1}, n_{2}, n_{3}}^{p_{1}, p_{2}, p_{3}}=(-1)^{p_{1}+p_{2}+p_{3}+1} C_{n_{2}, n_{1}, n_{3}}^{p_{1}, p_{3}, p_{2}}=(-1)^{p_{1}+p_{2}+p_{3}+1} C_{n_{1}, n_{3}, n_{2}}^{p_{3}, p_{2}, p_{1}}=(-1)^{p_{1}+p_{2}+p_{3}+1} C_{n_{3}, n_{2}, n_{1}}^{p_{2}, p_{1}, p_{3}}, \\
\sum_{k_{1}=0}^{p_{1}} \sum_{k_{2}=0}^{p_{2}} \sum_{k_{3}=0}^{p_{3}}\left(\begin{array}{l}
p_{1} \\
k_{1}
\end{array}\right)\left(\begin{array}{l}
p_{2} \\
k_{2}
\end{array}\right)\left(\begin{array}{l}
p_{3} \\
k_{3}
\end{array}\right)(-1)^{k_{1}+k_{2}+k_{3}} C_{n_{1}+p_{1}+k_{3}-k_{1}, n_{2}+p_{2}+k_{1}-k_{2}, n_{3}+p_{3}+k_{2}-k_{3}}^{p_{1} p_{3}}=C_{n_{1}, n_{2}, n_{3}}^{0,0,0} .
\end{gathered}
$$

The relevant building blocks for the quartic order in $b_{n_{i}}$ are $C_{n_{1}, n_{2}, n_{3}}^{3,3,3}$. We restrict to $n_{1} \leq n_{2} \leq n_{3}$ thanks to the first line in (A.10). 
- The coefficients with $n_{3}-3 \leq n_{2}$ and with $n_{2}-3 \leq n_{1}$ are undetermined by the recursion relations and we can set them to zero for simplicity.

- The coefficients with $n_{3}>n_{2}+3>n_{1}+6$ are generated through (A.10) after shifting $n_{2} \rightarrow n_{2}-3, n_{3} \rightarrow n_{3}-6$

$$
\begin{aligned}
C_{n_{1}, n_{2}, n_{3}}^{3,3,3}= & C_{n_{1}, n_{2}-3, n_{3}-6}^{0,0,0}+3 C_{n_{1}, n_{2}+1, n_{3}-1}^{3,3,}-3 C_{n_{1}, n_{2}+2, n_{3}-2}^{3,3,}+C_{n_{1}, n_{2}+3, n_{3}-3}^{3,3,3} \\
& +3 C_{n_{1}+1, n_{2}-1, n_{3}}^{3,3,3}-6 C_{n_{1}+1, n_{2}, n_{3}-1}^{3,3,3}+6 C_{n_{1}+1, n_{2}+2, n_{3}-3}^{3,3,3}-3 C_{n_{1}+1, n_{2}+3, n_{3}-4}^{3,3,3} \\
& -3 C_{n_{1}+2,3, n_{2}-2, n_{3}}^{3,3,}+15 C_{n_{1}+2, n_{2}, n_{3}-2}^{3,3,3}-15 C_{n_{1}+2,3, n_{2}+1, n_{3}-3}^{3,3}+3 C_{n_{1}+2,3, n_{2}+3, n_{3}-5}^{3,3,3} \\
& +C_{n_{1}+3, n_{2}-3, n_{3}}^{3,3,3}+6 C_{n_{1}+3, n_{2}-2, n_{3}-1}^{3,3,3}-15 C_{n_{1}+3, n_{2}-1, n_{3}-2}^{3,3,3}+15 C_{n_{1}+3, n_{2}+1, n_{3}-4}^{3,3,3} \\
& -6 C_{n_{1}+3, n_{2}+2, n_{3}-5}^{3,3,3}-C_{n_{1}+3, n_{2}+3, n_{3}-6}^{3,3,3}-3 C_{n_{1}+4, n_{2}-3, n_{3}-1}^{3,3,3}+15 C_{n_{1}+4, n_{2}-1, n_{3}-3}^{3,3,3} \\
& -15 C_{n_{1}+4, n_{2}, n_{3}-4}^{3,3,3}+3 C_{n_{1}+4, n_{2}+2, n_{3}-6}^{3,3,3}+3 C_{n_{1}+5, n_{2}-3, n_{3}-2}^{3,3,3}-6 C_{n_{1}+5, n_{2}-2, n_{3}-3}^{3,3,3} \\
& +6 C_{n_{1}+5, n_{2}, n_{3}-5}^{3,3,3}-3 C_{n_{1}+5, n_{2}+1, n_{3}-6}^{3,3,3}-C_{n_{1}+6, n_{2}-3, n_{3}-3}^{3,3,3}+3 C_{n_{1}+6, n_{2}-2, n_{3}-4}^{3,3,3} \\
& -3 C_{n_{1}+6, n_{2}-1, n_{3}-5}^{3,3,3}+C_{n_{1}+6, n_{2}, n_{3}-6}^{3,3,3} .
\end{aligned}
$$

\section{A.3 Two-loop ladder diagrams}

The quadruple ordered integral in the ladder contribution to (3.11) decomposes into a sum of

$$
D_{n_{1}, n_{2}, n_{3}, n_{4}}^{p_{1}, p_{2}}=\int_{0}^{2 \pi} d \theta_{1} \int_{0}^{\theta_{1}} d \theta_{2} \int_{0}^{\theta_{2}} d \theta_{3} \int_{0}^{\theta_{3}} d \theta_{4} \frac{e^{i\left(n_{1} \theta_{1}+n_{2} \theta_{2}+n_{3} \theta_{3}+n_{4} \theta_{4}\right)}}{\left(e^{i \theta_{1}}-e^{i \theta_{3}}\right)^{p_{1}}\left(e^{i \theta_{2}}-e^{i \theta_{4}}\right)^{p_{2}}} .
$$

The simplest integral of the family

$$
\begin{aligned}
D_{n_{1}, n_{2}, n_{3}, n_{4}}^{0,0}= & \frac{1}{n_{1}\left(n_{1}+n_{2}\right)\left(n_{1}+n_{2}+n_{3}\right)\left(n_{1}+n_{2}+n_{3}+n_{4}\right)}-\frac{e^{2 \pi i n_{1}}}{n_{1} n_{2}\left(n_{2}+n_{3}\right)\left(n_{2}+n_{3}+n_{4}\right)} \\
& +\frac{e^{2 \pi i\left(n_{1}+n_{2}\right)}}{n_{2} n_{3}\left(n_{1}+n_{2}\right)\left(n_{3}+n_{4}\right)}-\frac{e^{2 \pi i\left(n_{1}+n_{2}+n_{3}\right)}}{n_{3} n_{4}\left(n_{2}+n_{3}\right)\left(n_{1}+n_{2}+n_{3}\right)} \\
& +\frac{e^{2 \pi i\left(n_{1}+n_{2}+n_{3}+n_{4}\right)}}{n_{4}\left(n_{3}+n_{4}\right)\left(n_{2}+n_{3}+n_{4}\right)\left(n_{1}+n_{2}+n_{3}+n_{4}\right)}
\end{aligned}
$$

is non-singular for any integer $n_{i}$, with the appropriate application of L'Hôpital's rule, as before. We define the others through the relations

$$
\sum_{k_{1}=0}^{p_{1}} \sum_{k_{2}=0}^{p_{2}}\left(\begin{array}{l}
p_{1} \\
k_{1}
\end{array}\right)\left(\begin{array}{l}
p_{2} \\
k_{2}
\end{array}\right)(-1)^{k_{1}+k_{2}} D_{n_{1}+p_{1}-k_{1}, n_{2}+p_{2}-k_{2}, n_{3}+k_{1}, n_{4}+k_{2}}^{p_{1}, p_{2}}=D_{n_{1}, n_{2}, n_{3}, n_{4}}^{0,0}
$$

The ordered integrations (A.12) imply no parity for the integrals, which means that more recurrence formulas are needed to cover all values of $n_{i}$. We start with the solution for $D_{n_{1}, n_{2}, n_{3}, n_{4}}^{2,2}$.

- The coefficients with $0 \leq n_{3}-n_{1} \leq 3$ or $0 \leq n_{2} \leq 1$ are arbitrary constants. 
- The coefficients with $4 \leq n_{3}-n_{1}$ and $2 \leq n_{2}$ are given by

$$
\begin{aligned}
D_{n_{1}, n_{2}, n_{3}, n_{4}}^{2,2}= & D_{n_{1}, n_{2}-2, n_{3}-2, n_{4}}^{0,0}-D_{n_{1}, n_{2}-2, n_{3}, n_{4}+2}^{2,2}+2 D_{n_{1}, n_{2}-1, n_{3}, n_{4}+1}^{2,2} \\
& +2 D_{n_{1}+1, n_{2}-2, n_{3}-1, n_{4}+2}^{2,2}-4 D_{n_{1}+1, n_{2}-1, n_{3}-1, n_{4}+1}^{2,2}+2 D_{n_{1}+1, n_{2}, n_{3}-1, n_{4}}^{2,2} \\
& -D_{n_{1}+2, n_{2}-2, n_{3}-2, n_{4}+2}^{2,2}+2 D_{n_{1}+2, n_{2}-1, n_{3}-2, n_{4}+1}^{2,2}-D_{n_{1}+2, n_{2}, n_{3}-2, n_{4}}^{2,2}
\end{aligned}
$$

- For $n_{3}-n_{1} \leq-1$ and $2 \leq n_{2}$ we find

$$
\begin{aligned}
D_{n_{1}, n_{2}, n_{3}, n_{4}}^{2,2}= & D_{n_{1}-2, n_{2}-2, n_{3}, n_{4}}^{0,0}-D_{n_{1}-2, n_{2}-2, n_{3}+2, n_{4}+2}^{2,2}+2 D_{n_{1}-2, n_{2}-1, n_{3}+2, n_{4}+1}^{2,2} \\
& -D_{n_{1}-2, n_{2}, n_{3}+2, n_{4}}^{2,2}+2 D_{n_{1}-1, n_{2}-2, n_{3}+1, n_{4}+2}^{2,2}-4 D_{n_{1}-1, n_{2}-1, n_{3}+1, n_{4}+1}^{2,2} \\
& +2 D_{n_{1}-1, n_{2}, n_{3}+1, n_{4}}^{2,2}-D_{n_{1}, n_{2}-2, n_{3}, n_{4}+2}^{2,2}+2 D_{n_{1}, n_{2}-1, n_{3}, n_{4}+1}^{2,2}
\end{aligned}
$$

- For $n_{3}-n_{1} \leq-1$ and $n_{2} \leq-1$ we find

$$
\begin{aligned}
D_{n_{1}, n_{2}, n_{3}, n_{4}}^{2,2}= & D_{n_{1}-2, n_{2}, n_{3}, n_{4}-2}^{0,0}-D_{n_{1}-2, n_{2}, n_{3}+2, n_{4}}^{2,2}+2 D_{n_{1}-2, n_{2}+1, n_{3}+2, n_{4}-1}^{2,2} \\
& -D_{n_{1}-2, n_{2}+2, n_{3}+2, n_{4}-2}^{2,2}+2 D_{n_{1}-1, n_{2}, n_{3}+1, n_{4}}^{2,2}-4 D_{n_{1}-1, n_{2}+1, n_{3}+1, n_{4}-1}^{2,2} \\
& +2 D_{n_{1}-1, n_{2}+2, n_{3}+1, n_{4}-2}^{2,2}+2 D_{n_{1}, n_{2}+1, n_{3}, n_{4}-1}^{2,2}-D_{n_{1}, n_{2}+2, n_{3}, n_{4}-2}^{2,2} .
\end{aligned}
$$

- Finally, the coefficients with $4 \leq n_{3}-n_{1}$ and $n_{2} \leq-1$ are given by

$$
\begin{aligned}
D_{n_{1}, n_{2}, n_{3}, n_{4}}^{2,2}= & D_{n_{1}, n_{2}, n_{3}-2, n_{4}-2}^{0,0}+2 D_{n_{1}, n_{2}+1, n_{3}, n_{4}-1}^{2,2}-D_{n_{1}, n_{2}+2, n_{3}, n_{4}-2}^{2,2} \\
& +2 D_{n_{1}+1, n_{2}, n_{3}-1, n_{4}}^{2,2}-4 D_{n_{1}+1, n_{2}+1, n_{3}-1, n_{4}-1}^{2,2}+2 D_{n_{1}+1, n_{2}+2, n_{3}-1, n_{4}-2}^{2,2} \\
& -D_{n_{1}+2, n_{2}, n_{3}-2, n_{4}}^{2,2}+2 D_{n_{1}+2, n_{2}+1, n_{3}-2, n_{4}-1}^{2,2}-D_{n_{1}+2, n_{2}+2, n_{3}-2, n_{4}-2}^{2,2}
\end{aligned}
$$

The order $\lambda^{2} \epsilon^{4}$ depends also on the elementary integrals $D_{n_{1}, n_{2}, n_{3}, n_{4}}^{4,4}$.

- The coefficients with $0 \leq n_{3}-n_{1} \leq 7$ or $0 \leq n_{2} \leq 3$ are arbitrary constants.

- The coefficients with $8 \leq n_{3}-n_{1}$ and $4 \leq n_{2}$ are generated recursively by

$$
\begin{aligned}
D_{n_{1}, n_{2}, n_{3}, n_{4}}^{4,4}= & D_{n_{1}, n_{2}-4, n_{3}-4, n_{4}}^{0,0}-D_{n_{1}, n_{2}-4, n_{3}, n_{4}+4}^{4,4}+4 D_{n_{1}, n_{2}-3, n_{3}, n_{4}+3}^{4,4}-6 D_{n_{1}, n_{2}-2, n_{3}, n_{4}+2}^{4,4} \\
& +4 D_{n_{1}, n_{2}-1, n_{3}, n_{4}+1}^{4,4}+4 D_{n_{1}+1, n_{2}-4, n_{3}-1, n_{4}+4}^{4,4}-16 D_{n_{1}+1, n_{2}-3, n_{3}-1, n_{4}+3}^{4,4} \\
& +24 D_{n_{1}+1, n_{2}-2, n_{3}-1, n_{4}+2}^{4,4}-16 D_{n_{1}+1, n_{2}-1, n_{3}-1, n_{4}+1}^{4,4}+4 D_{n_{1}+1, n_{2}, n_{3}-1, n_{4}}^{4,4} \\
& -6 D_{n_{1}+2, n_{2}-4, n_{3}-2, n_{4}+4}^{4,4}+24 D_{n_{1}+2, n_{2}-3, n_{3}-2, n_{4}+3}^{4,4}-36 D_{n_{1}+2, n_{2}-2, n_{3}-2, n_{4}+2}^{4,4} \\
& +24 D_{n_{1}+2, n_{2}-1, n_{3}-2, n_{4}+1}^{4,4}-6 D_{n_{1}+2, n_{2}, n_{3}-2, n_{4}}^{4,4}+4 D_{n_{1}+3, n_{2}-4, n_{3}-3, n_{4}+4}^{4,4} \\
& -16 D_{n_{1}+3, n_{2}-3, n_{3}-3, n_{4}+3}^{4,4}+24 D_{n_{1}+3, n_{2}-2, n_{3}-3, n_{4}+2}^{4,4}-16 D_{n_{1}+3, n_{2}-1, n_{3}-3, n_{4}+1}^{4,4} \\
& +4 D_{n_{1}+3, n_{2}, n_{3}-3, n_{4}}^{4,4}-D_{n_{1}+4, n_{2}-4, n_{3}-4, n_{4}+4}^{4,4}+4 D_{n_{1}+4, n_{2}-3, n_{3}-4, n_{4}+3}^{4,4} \\
& -6 D_{n_{1}+4, n_{2}-2, n_{3}-4, n_{4}+2}^{4,4}+4 D_{n_{1}+4, n_{2}-1, n_{3}-4, n_{4}+1}^{4,4}-D_{n_{1}+4, n_{2}, n_{3}-4, n_{4}}^{4,4} \quad \text { (A.19) }
\end{aligned}
$$


- The coefficients with $n_{3}-n_{1} \leq-1$ and $4 \leq n_{2}$ are generated recursively by

$$
\begin{aligned}
D_{n_{1}, n_{2}, n_{3}, n_{4}}^{4,4}= & D_{n_{1}-4, n_{2}-4, n_{3}, n_{4}}^{0,0}-D_{n_{1}-4, n_{2}-4, n_{3}+4, n_{4}+4}^{4,4}+4 D_{n_{1}-4, n_{2}-3, n_{3}+4, n_{4}+3}^{4,4} \\
& -6 D_{n_{1}-4, n_{2}-2, n_{3}+4, n_{4}+2}^{4,4}+4 D_{n_{1}-4, n_{2}-1, n_{3}+4, n_{4}+1}^{4,4}-D_{n_{1}-4, n_{2}, n_{3}+4, n_{4}}^{4,4} \\
& +4 D_{n_{1}-3, n_{2}-4, n_{3}+3, n_{4}+4}^{4,4}-16 D_{n_{1}-3, n_{2}-3, n_{3}+3, n_{4}+3}^{4,4}+24 D_{n_{1}-3, n_{2}-2, n_{3}+3, n_{4}+2}^{4,4} \\
& -16 D_{n_{1}-3, n_{2}-1, n_{3}+3, n_{4}+1}^{4,4}+4 D_{n_{1}-3, n_{2}, n_{3}+3, n_{4}}^{4,4}-6 D_{n_{1}-2, n_{2}-4, n_{3}+2, n_{4}+4}^{4,4} \\
& +24 D_{n_{1}-2, n_{2}-3, n_{3}+2, n_{4}+3}^{4,4}-36 D_{n_{1}-2, n_{2}-2, n_{3}+2, n_{4}+2}^{4,4}+24 D_{n_{1}-2, n_{2}-1, n_{3}+2, n_{4}+1}^{4,4} \\
& -6 D_{n_{1}-2, n_{2}, n_{3}+2, n_{4}}^{4,4}+4 D_{n_{1}-1, n_{2}-4, n_{3}+1, n_{4}+4}^{4,4}-16 D_{n_{1}-1, n_{2}-3, n_{3}+1, n_{4}+3}^{4,4} \\
& +24 D_{n_{1}-1, n_{2}-2, n_{3}+1, n_{4}+2}^{4,4}-16 D_{n_{1}-1, n_{2}-1, n_{3}+1, n_{4}+1}^{4,4}+4 D_{n_{1}-1, n_{2}, n_{3}+1, n_{4}}^{4,4} \\
& -D_{n_{1}, n_{2}-4, n_{3}, n_{4}+4}^{4,4}+4 D_{n_{1}, n_{2}-3, n_{3}, n_{4}+3}^{4,4}-6 D_{n_{1}, n_{2}-2, n_{3}, n_{4}+2}^{4,4}+4 D_{n_{1}, n_{2}-1, n_{3}, n_{4}+1}^{4,4}
\end{aligned}
$$

- The coefficients with $n_{3}-n_{1} \leq-1$ and $n_{2} \leq-1$ are generated recursively by

$$
\begin{aligned}
D_{n_{1}, n_{2}, n_{3}, n_{4}}^{4,4}= & D_{n_{1}-4, n_{2}, n_{3}, n_{4}-4}^{0,0}-D_{n_{1}-4, n_{2}, n_{3}+4, n_{4}}^{4,4}+4 D_{n_{1}-4, n_{2}+1, n_{3}+4, n_{4}-1}^{4,4} \\
& -6 D_{n_{1}-4, n_{2}+2, n_{3}+4, n_{4}-2}^{4,4}+4 D_{n_{1}-4, n_{2}+3, n_{3}+4, n_{4}-3}^{4,4}-D_{n_{1}-4, n_{2}+4, n_{3}+4, n_{4}-4}^{4,4} \\
& +4 D_{n_{1}-3, n_{2}, n_{3}+3, n_{4}}^{4,4}-16 D_{n_{1}-3, n_{2}+1, n_{3}+3, n_{4}-1}^{4,4}+24 D_{n_{1}-3, n_{2}+2, n_{3}+3, n_{4}-2}^{4,4} \\
& -16 D_{n_{1}-3, n_{2}+3, n_{3}+3, n_{4}-3}^{4,4}+4 D_{n_{1}-3, n_{2}+4, n_{3}+3, n_{4}-4}^{4,4}-6 D_{n_{1}-2, n_{2}, n_{3}+2, n_{4}}^{4,4} \\
& +24 D_{n_{1}-2, n_{2}+1, n_{3}+2, n_{4}-1}^{4,4}-36 D_{n_{1}-2, n_{2}+2, n_{3}+2, n_{4}-2}^{4,4}+24 D_{n_{1}-2, n_{2}+3, n_{3}+2, n_{4}-3}^{4,4} \\
& -6 D_{n_{1}-2, n_{2}+4, n_{3}+2, n_{4}-4}^{4,4}+4 D_{n_{1}-1, n_{2}, n_{3}+1, n_{4}}^{4,4}-16 D_{n_{1}-1, n_{2}+1, n_{3}+1, n_{4}-1}^{4,4} \\
& +24 D_{n_{1}-1, n_{2}+2, n_{3}+1, n_{4}-2}^{4,4}-16 D_{n_{1}-1, n_{2}+3, n_{3}+1, n_{4}-3}^{4,4}+4 D_{n_{1}-1, n_{2}+4, n_{3}+1, n_{4}-4}^{4,4} \\
& +4 D_{n_{1}, n_{2}+1, n_{3}, n_{4}-1}^{4,4}-6 D_{n_{1}, n_{2}+2, n_{3}, n_{4}-2}^{4,4}+4 D_{n_{1}, n_{2}+3, n_{3}, n_{4}-3}^{4,4}-D_{n_{1}, n_{2}+4, n_{3}, n_{4}-4}^{4,4}
\end{aligned}
$$

- The coefficients with $8 \leq n_{3}-n_{1}$ and $n_{2} \leq-1$ are generated recursively by

$$
\begin{aligned}
D_{n_{1}, n_{2}, n_{3}, n_{4}}^{4,4}= & D_{n_{1}, n_{2}, n_{3}-4, n_{4}-4}^{0,0}+4 D_{n_{1}, n_{2}+1, n_{3}, n_{4}-1}^{4,4}-6 D_{n_{1}, n_{2}+2, n_{3}, n_{4}-2}^{4,4}+4 D_{n_{1}, n_{2}+3, n_{3}, n_{4}-3}^{4,4} \\
& -D_{n_{1}, n_{2}+4, n_{3}, n_{4}-4}^{4,4}+4 D_{n_{1}+1, n_{2}, n_{3}-1, n_{4}}^{4,4}-16 D_{n_{1}+1, n_{2}+1, n_{3}-1, n_{4}-1}^{4,4} \\
& +24 D_{n_{1}+1, n_{2}+2, n_{3}-1, n_{4}-2}^{4,4}-16 D_{n_{1}+1, n_{2}+3, n_{3}-1, n_{4}-3}^{4,4}+4 D_{n_{1}+1, n_{2}+4, n_{3}-1, n_{4}-4}^{4,4} \\
& -6 D_{n_{1}+2, n_{2}, n_{3}-2, n_{4}}^{4,4}+24 D_{n_{1}+2, n_{2}+1, n_{3}-2, n_{4}-1}^{4,4}-36 D_{n_{1}+2, n_{2}+2, n_{3}-2, n_{4}-2}^{4,4} \\
& +24 D_{n_{1}+2, n_{2}+3, n_{3}-2, n_{4}-3}^{4,4}-6 D_{n_{1}+2, n_{2}+4, n_{3}-2, n_{4}-4}^{4,4}+4 D_{n_{1}+3, n_{2}, n_{3}-3, n_{4}}^{4,4} \\
& -16 D_{n_{1}+3, n_{2}+1, n_{3}-3, n_{4}-1}^{4,4}+24 D_{n_{1}+3, n_{2}+2, n_{3}-3, n_{4}-2}^{4,4}-16 D_{n_{1}+3, n_{2}+3, n_{3}-3, n_{4}-3}^{4,4} \\
& +4 D_{n_{1}+3, n_{2}+4, n_{3}-3, n_{4}-4}^{4,4} D_{n_{1}+4, n_{2}, n_{3}-4, n_{4}}^{4,4}+4 D_{n_{1}+4, n_{2}+1, n_{3}-4, n_{4}-1}^{4,4} \\
& -6 D_{n_{1}+4, n_{2}+2, n_{3}-4, n_{4}-2}^{4,4}+4 D_{n_{1}+4, n_{2}+3, n_{3}-4, n_{4}-3}^{4,4}-D_{n_{1}+4, n_{2}+4, n_{3}-4, n_{4}-4}^{4,4}
\end{aligned}
$$

Open Access. This article is distributed under the terms of the Creative Commons Attribution License (CC-BY 4.0), which permits any use, distribution and reproduction in any medium, provided the original author(s) and source are credited. 


\section{References}

[1] J.K. Erickson, G.W. Semenoff and K. Zarembo, Wilson loops in $\mathcal{N}=4$ supersymmetric Yang-Mills theory, Nucl. Phys. B 582 (2000) 155 [hep-th/0003055] [INSPIRE].

[2] N. Drukker and D.J. Gross, An exact prediction of $\mathcal{N}=4$ SUSYM theory for string theory, J. Math. Phys. 42 (2001) 2896 [hep-th/0010274] [INSPIRE].

[3] V. Pestun, Localization of gauge theory on a four-sphere and supersymmetric Wilson loops, Commun. Math. Phys. 313 (2012) 71 [arXiv:0712.2824] [INSPIRE].

[4] R. Ishizeki, M. Kruczenski and S. Ziama, Notes on euclidean Wilson loops and Riemann theta functions, Phys. Rev. D 85 (2012) 106004 [arXiv:1104.3567] [INSPIRE].

[5] G.W. Semenoff and D. Young, Wavy Wilson line and AdS/CFT, Int. J. Mod. Phys. A 20 (2005) 2833 [hep-th/0405288] [inSPIRE].

[6] D. Correa, J. Henn, J. Maldacena and A. Sever, An exact formula for the radiation of a moving quark in $\mathcal{N}=4$ super Yang Mills, JHEP 06 (2012) 048 [arXiv:1202.4455] [INSPIRE].

[7] B. Fiol, B. Garolera and A. Lewkowycz, Exact results for static and radiative fields of a quark in $\mathcal{N}=4$ super Yang-Mills, JHEP 05 (2012) 093 [arXiv:1202.5292] [INSPIRE].

[8] M. Cooke, A. Dekel and N. Drukker, The Wilson loop CFT: Insertion dimensions and structure constants from wavy lines, J. Phys. A 50 (2017) 335401 [arXiv:1703.03812] [INSPIRE].

[9] S. Giombi, R. Roiban and A.A. Tseytlin, Half-BPS Wilson loop and AdS $S_{2} / C F T_{1}$, Nucl. Phys. B 922 (2017) 499 [arXiv: 1706.00756] [InSPIRE].

[10] M. Kim and N. Kiryu, Structure constants of operators on the Wilson loop from integrability, JHEP 11 (2017) 116 [arXiv:1706. 02989] [INSPIRE].

[11] M. Kim, N. Kiryu, S. Komatsu and T. Nishimura, Structure Constants of Defect Changing Operators on the 1/2 BPS Wilson Loop, JHEP 12 (2017) 055 [arXiv:1710.07325] [INSPIRE].

[12] K. Pohlmeyer, Integrable hamiltonian systems and interactions through quadratic constraints, Commun. Math. Phys. 46 (1976) 207 [InSPIRE].

[13] A. Dekel, Wilson loops and minimal surfaces beyond the wavy approximation, JHEP 03 (2015) 085 [arXiv: 1501.04202] [INSPIRE].

[14] T. Klose, F. Loebbert and H. Münkler, Master symmetry for holographic Wilson loops, Phys. Rev. D 94 (2016) 066006 [arXiv:1606.04104] [INSPIRE].

[15] T. Klose, F. Loebbert and H. Münkler, Nonlocal symmetries, spectral parameter and minimal surfaces in AdS/CFT, Nucl. Phys. B 916 (2017) 320 [arXiv:1610.01161] [INSPIRE].

[16] A. Bassetto, L. Griguolo, F. Pucci and D. Seminara, Supersymmetric Wilson loops at two loops, JHEP 06 (2008) 083 [arXiv:0804.3973] [INSPIRE].

[17] M. Kruczenski, Wilson loops and minimal area surfaces in hyperbolic space, JHEP 11 (2014) 065 [arXiv: 1406.4945] [INSPIRE].

[18] Y. He and M. Kruczenski, Minimal area surfaces in AdS $S_{3}$ through integrability, J. Phys. A 50 (2017) 495401 [arXiv: 1705.10037] [INSPIRE].

[19] C. Huang, Y. He and M. Kruczenski, Minimal area surfaces dual to Wilson loops and the Mathieu equation, JHEP 08 (2016) 088 [arXiv: 1604.00078] [INSPIRE]. 
[20] N. Drukker and S. Kawamoto, Small deformations of supersymmetric Wilson loops and open spin-chains, JHEP 07 (2006) 024 [hep-th/0604124] [INSPIRE].

[21] N. Drukker, Integrable Wilson loops, JHEP 10 (2013) 135 [arXiv:1203.1617] [INSPIRE].

[22] D. Correa, J. Maldacena and A. Sever, The quark anti-quark potential and the cusp anomalous dimension from a TBA equation, JHEP 08 (2012) 134 [arXiv:1203.1913] [INSPIRE].

[23] N. Gromov and A. Sever, Analytic solution of Bremsstrahlung TBA, JHEP 11 (2012) 075 [arXiv: 1207.5489] [INSPIRE].

[24] N. Gromov and F. Levkovich-Maslyuk, Quantum spectral curve for a cusped Wilson line in $\mathcal{N}=4$ SYM, JHEP 04 (2016) 134 [arXiv:1510.02098] [INSPIRE].

[25] P. Liendo and C. Meneghelli, Bootstrap equations for $\mathcal{N}=4$ SYM with defects, JHEP 01 (2017) 122 [arXiv: 1608.05126] [INSPIRE].

[26] A. Cavaglià, N. Gromov and F. Levkovich-Maslyuk, Quantum spectral curve and structure constants in $\mathcal{N}=4$ SYM: cusps in the ladder limit, JHEP 10 (2018) 060 [arXiv: 1802.04237] [INSPIRE].

[27] S. Giombi and S. Komatsu, Exact correlators on the Wilson loop in $\mathcal{N}=4 S Y M$ : Localization, defect CFT, and integrability, JHEP 05 (2018) 109 [Erratum ibid. 11 (2018) 123] [arXiv: 1802.05201] [INSPIRE].

[28] P. Liendo, C. Meneghelli and V. Mitev, Bootstrapping the half-BPS line defect, JHEP 10 (2018) 077 [arXiv: 1806.01862] [INSPIRE].

[29] S. Giombi and S. Komatsu, More exact results in the Wilson loop defect CFT: bulk-defect OPE, nonplanar corrections and quantum spectral curve, arXiv:1811.02369 [INSPIRE].

[30] J.C. Toledo, Smooth Wilson loops from the continuum limit of null polygons, arXiv: 1410.5896 [INSPIRE].

[31] Y. He, C. Huang and M. Kruczenski, Minimal area surfaces in $A d S_{n+1}$ and Wilson loops, JHEP 02 (2018) 027 [arXiv: 1712.06269] [INSPIRE].

[32] N. Drukker, J. Plefka and D. Young, Wilson loops in 3-dimensional $\mathcal{N}=6$ supersymmetric Chern-Simons Theory and their string theory duals, JHEP 11 (2008) 019 [arXiv:0809.2787] [INSPIRE].

[33] B. Chen and J.-B. Wu, Supersymmetric Wilson loops in $\mathcal{N}=6$ super Chern-Simons-matter theory, Nucl. Phys. B $\mathbf{8 2 5}$ (2010) 38 [arXiv:0809.2863] [INSPIRE].

[34] S.-J. Rey, T. Suyama and S. Yamaguchi, Wilson loops in superconformal Chern-Simons theory and fundamental strings in anti-de Sitter supergravity dual, JHEP 03 (2009) 127 [arXiv:0809.3786] [INSPIRE].

[35] N. Drukker and D. Trancanelli, A supermatrix model for $\mathcal{N}=6$ super Chern-Simons-matter theory, JHEP 02 (2010) 058 [arXiv: 0912.3006] [INSPIRE].

[36] S.-x. Chu, D. Hou and H.-c. Ren, The subleading term of the strong coupling expansion of the eeavy-quark potential in a $\mathcal{N}=4$ super Yang-Mills vacuum, JHEP 08 (2009) 004 [arXiv: 0905.1874] [INSPIRE].

[37] V. Forini, Quark-antiquark potential in AdS at one loop, JHEP 11 (2010) 079 [arXiv: 1009.3939] [INSPIRE]. 
[38] N. Drukker and V. Forini, Generalized quark-antiquark potential at weak and strong coupling, JHEP 06 (2011) 131 [arXiv: 1105.5144] [INSPIRE].

[39] N. Drukker, D.J. Gross and A.A. Tseytlin, Green-Schwarz string in $A d S_{5} \times S^{5}$ : Semiclassical partition function, JHEP 04 (2000) 021 [hep-th/0001204] [INSPIRE].

[40] V. Forini, V. Giangreco M. Puletti, L. Griguolo, D. Seminara and E. Vescovi, Precision calculation of 1/4-BPS Wilson loops in $A d S_{5} \times S^{5}$, JHEP 02 (2016) 105 [arXiv: 1512.00841] [INSPIRE].

[41] A. Faraggi, L.A. Pando Zayas, G.A. Silva and D. Trancanelli, Toward precision holography with supersymmetric Wilson loops, JHEP 04 (2016) 053 [arXiv: 1601.04708] [INSPIRE].

[42] V. Forini, A.A. Tseytlin and E. Vescovi, Perturbative computation of string one-loop corrections to Wilson loop minimal surfaces in $A d S_{5} \times S^{5}$, JHEP 03 (2017) 003 [arXiv: 1702.02164] [INSPIRE].

[43] A. Cagnazzo, D. Medina-Rincon and K. Zarembo, String corrections to circular Wilson loop and anomalies, JHEP 02 (2018) 120 [arXiv:1712.07730] [INSPIRE].

[44] D. Medina-Rincon, A.A. Tseytlin and K. Zarembo, Precision matching of circular Wilson loops and strings in $A d S_{5} \times S^{5}$, JHEP 05 (2018) 199 [arXiv: 1804.08925] [INSPIRE]. 